\title{
APPLYING MODERN MOLECULAR TECHNOLOGIES IN SUPPORT OF THE STERILE INSECT TECHNIQUE
}

\author{
I. HÄCKER ${ }^{1,2}$, K. BOURTZIS ${ }^{3}$ AND M. F. SCHETELIG ${ }^{1,2}$ \\ ${ }^{1}$ Institute for Insect Biotechnology, Department for Insect Biotechnology in Plant \\ Protection, Justus-Liebig-University Giessen, 35394 Giessen, Germany \\ Email:Marc.Schetelig@agrar.uni-giessen.de \\ ${ }^{2}$ Fraunhofer Institute for Molecular Biology and Applied Ecology (IME), Division of \\ Bioresources, Dept. of Insect Pest and Vector Control, 35394 Giessen, Germany \\ ${ }^{3}$ Joint FAO/IAEA Division of Nuclear Techniques in Food and Agriculture, \\ International Atomic Energy Agency, A-1400 Vienna, Austria
}

\section{TABLE OF CONTENTS}

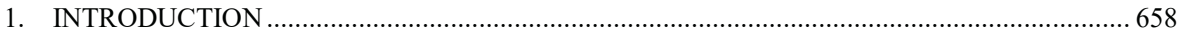

2. KEY MOLECULAR TECHNOLOGIES FOR POPULATION CONTROL ..................................6 661

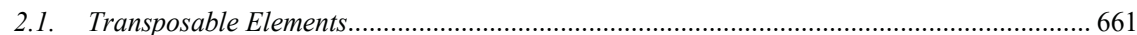

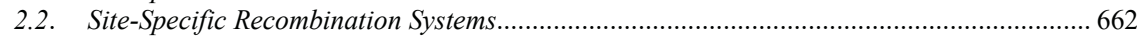

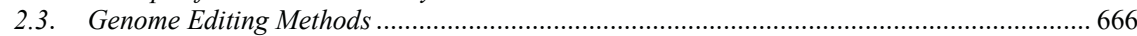

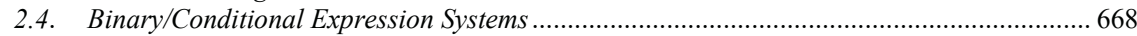

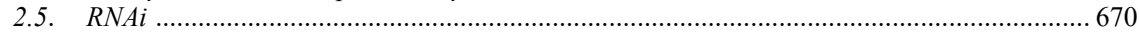

3. APPLYING MOLECULAR TECHNOLOGIES TO IMPROVE THE SIT ...............................672

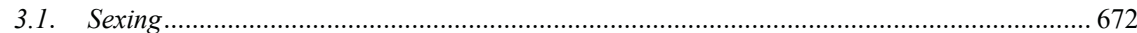

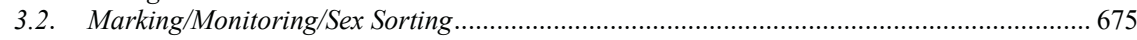

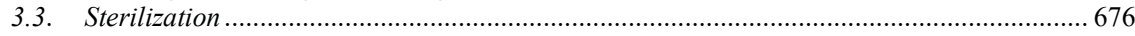

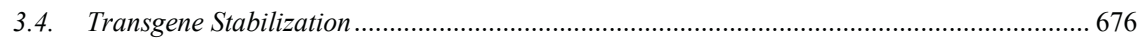

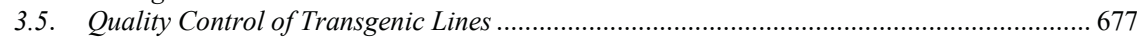

4. SUCCESSFULLY APPLYING MOLECULAR TOOLS - FIRST RELEASES OF

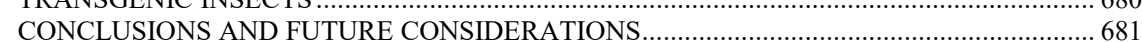

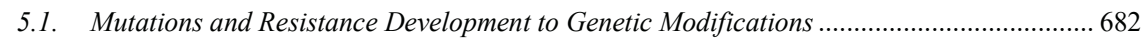

5.2. Regulatory Considerations for the Application of Transgenic Insects............................... 684

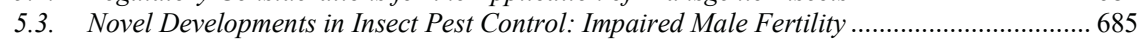

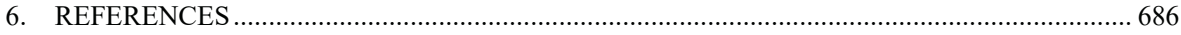




\section{SUMMARY}

The accelerating development of modern biotechnology, resulting continuously in new molecular techniques, has significantly increased the potential of understanding and modifying the genetic information in various organisms, from mammals to many insect species. Key molecular techniques developed over the past three decades include classical transformation technologies via transposable elements, and site-specific genome modification techniques using recombinases, both permitting the introduction of new genes or regulatory elements into the genome and their subsequent modification or (partial) removal. Tools like TALEN or CRISPR permit the precise editing of genomes at pre-defined positions, ranging from single-base changes to the knockout of complete genes, or the introduction of exogenous genetic material. On the other hand, RNA interference (RNAi) does not change genomic information but instead influences gene expression levels to various degrees, including complete suppression of expression. All of these tools can be used to develop or refine insect strains to improve the efficiency of existing pest control programmes, or to develop new SIT-based control systems. Modern biotechnology tools are being exploited to develop self-limiting as well as self-sustaining approaches. While the latter aim at population replacement strategies using gene drive or Wolbachia-based approaches and are mostly developed for vector and disease control, SIT-based control systems are self-limiting strategies that are supposed to leave no ecological footprint. Molecular technologies have already been applied to create sterility and sexing strains, and to introduce stable markers for monitoring in several insect species including the Mediterranean fruit fly, Mexican fruit fly, Australian sheep blow fly, New World screwworm, pink bollworm, and various mosquito species. Moreover, several of these strains have undergone an initial evaluation under mass-rearing scenarios or in open-field trials, although their wider adoption has so far been slow in view of low public acceptance of transgenic approaches, and the regulatory requirements and approvals that are required in most countries for their application.

\section{INTRODUCTION}

Successful implementation of the sterile insect technique (SIT) for insect pest control depends on several key factors, e.g. mass-rearing and irradiating the target pest insect. Both procedures can result in reduced biological quality and/or mating competitiveness of the produced sterile insects (Hooper 1972; Bloem et al. 2004; Andreasen and Curtis 2005; Parker and Mehta 2007; Guerfali et al. 2011). Besides sterilization by irradiation, it has been shown that sometimes other sterilization strategies, such as transgenic or symbiont-based approaches, also come at a cost to the mating competitiveness of the sterilized males (Catteruccia et al. 2003; Irvin et al. 2004; Bourtzis et al. 2014; Häcker et al. 2017; Kyritsis et al. 2019). This reduction in biological quality and mating competitiveness of the produced sterile insects influences the efficacy of SIT projects, and thereby their cost and feasibility. However, it should be noted that recent developments on insect symbiosis have shown that probiotic bacterial applications may significantly improve the biological quality (including male mating competitiveness) of the sterile insects (Ben Ami et al. 2010; Hamden et al. 2013; Ras et al. 2017; Augustinos et al., this volume).

Another important and challenging factor is the efficient and robust sex separation (sexing) for male-only releases. In the case of agricultural pests, this may affect the efficiency in action as well as the economy in production (Hendrichs et al. 1995; Cáceres et al. 2004). However, in the case of vector species such as mosquitoes, male-only releases are a prerequisite for SIT applications since females bite, blood-feed, and transmit major human pathogens. Therefore, efficient sexing on a large scale is needed (Gilles et al. 2014; Parker, Mamai et al., this volume).

For some insect pest species, morphological traits or markers have been developed in the last decades that help to discriminate between sterile and wild 
insects in the target area, and in particular to separate sexes. This is the case for the naturally occurring pupal size difference between males and females in some Aedes mosquitoes, which is being exploited for mechanical sexing (Bellini et al. 2018; Zacarés et al. 2018), although it is difficult to achieve a 100\% pure separation and is very labour-intensive when applied at larger scales. Genetic sexing strains (GSS) (Franz et al., this volume) offer the possibility of efficient and large-scale sexing. This has been achieved for several species including Anastrepha ludens (Loew) (Orozco et al. 2013), Bactrocera carambolae Drew and Hancock (Isasawin et al. 2014), Bactrocera dorsalis (Hendel) (Isasawin et al. 2012), Ceratitis capitata (Wiedemann) (Franz 1995), Anopheles albimanus Wiedemann (Kaiser et al. 1978), Anopheles arabiensis Patton (Yamada et al. 2012), Aedes albopictus (Skuse) (Lebon et al. 2018), and Zeugodacus cucurbitae (Coquillett) (McInnis et al. 2004), and for some species is successfully being used for sexing under mass-rearing conditions in current SIT programmes, while being further developed for others.

These genetic GSS were created by mutagenesis and classical genetics, and are based on two components: a mutation that can be used as a selectable recessive marker, and the placement of the dominant wild-type allele of this marker on the Ychromosome that links it to the male sex. Thus, the males are heterozygous for the recessive mutation and therefore are phenotypically wild type, while in homozygous females, the mutation can be used for sorting or killing the females. However, genetic sexing systems produced by mutagenesis and classical genetics in one species might not be transferable to another species, as the underlying mutation is often a random product. Molecular technologies offer new perspectives and strategies to create insect strains possessing the traits needed for an effective pest control programme (Häcker and Schetelig 2018). These modern biotechnologies involve transgenic as well as non-transgenic approaches, and can be applied to optimize existing, or develop new, strategies and strains, thereby complementing classical approaches. Molecular technologies can be used either as stand-alone techniques, e.g. sexing systems for population suppression (Häcker and Schetelig 2018), or could be combined with the SIT and/or the incompatible insect technique (IIT) (Bourtzis et al. 2016; Lees et al., this volume), to improve efficiency and to increase the range of pest insects targeted by pest control strategies that are environment-friendly. Moreover, once the mechanism of a classical GSS is uncovered, molecular technologies could help build similar non-transgenic sexing strains in other species.

In support of and as an alternative to classical genetic approaches, molecular technologies and IIT approaches are being exploited to create population suppression, sexing, or marking systems in several insect pest species. The current key molecular technologies that can be used to support the SIT, including sitespecific recombination technologies, genome editing tools, and RNA interference (RNAi), are described below. Insect transformation via transposable elements is also included; it is still a major tool to produce transgenic insects in spite of the fast development and adaptation of new biotechnologies, which may help address the needs of population suppression programmes against major insect pest species effectively and in a relatively short period of time. Progress made in developing such molecular technologies for use in the SIT is described, including their 
preliminary applications in the first field trials, as well as the regulations and public acceptance issues that must be considered when transgenic insects are involved.

To assist in understanding the terminology used in this chapter, some definitions are given in Box 1 .

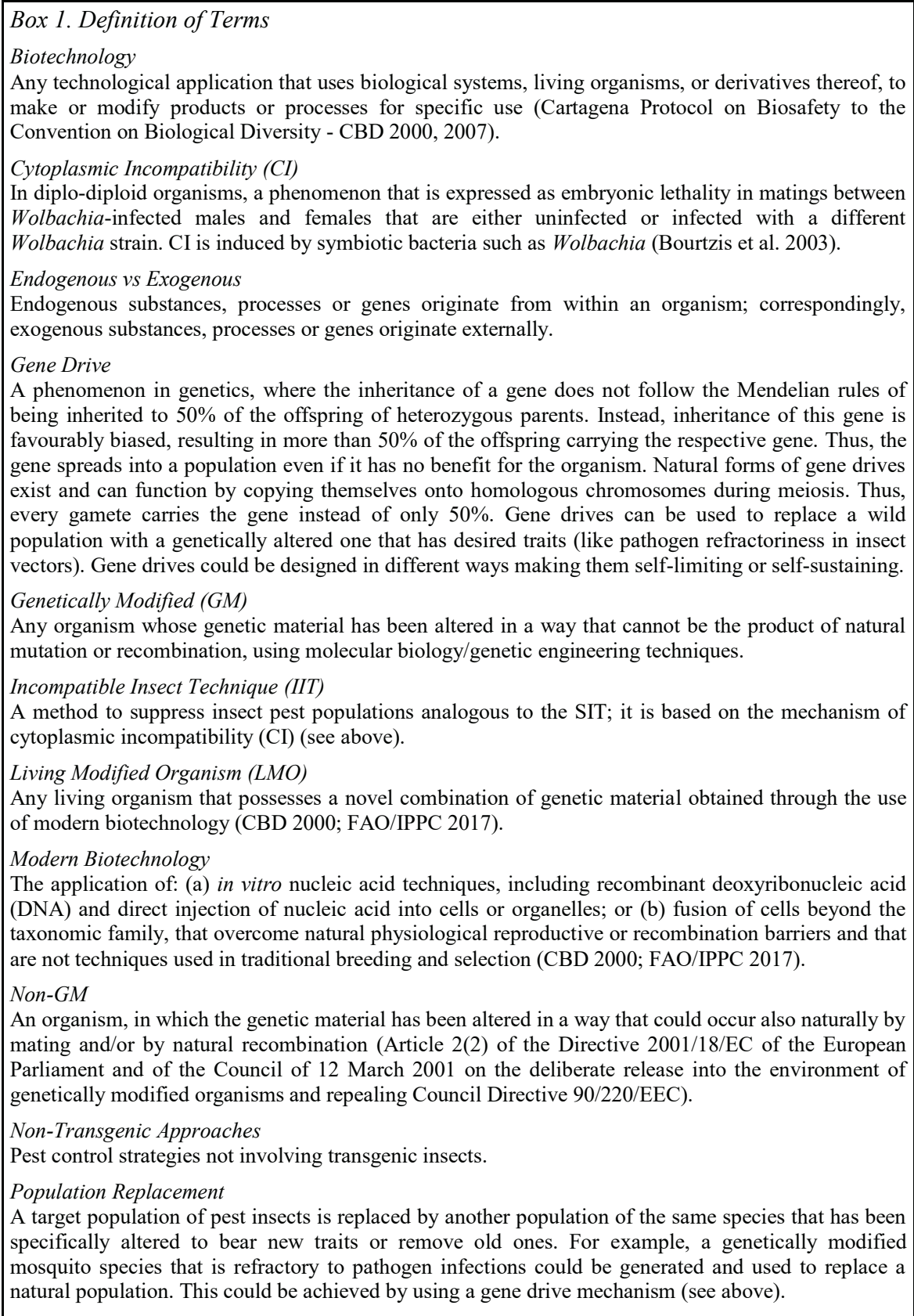




\begin{abstract}
Box 1. Definition of Terms -- Continued
RNA Interference

A conserved biological process to suppress gene expression or translation by neutralizing the corresponding messenger RNA (mRNA) molecules. Two types of small RNA molecules, microRNAs (miRNAs) and small interfering RNAs (siRNAs) in combination with several protein factors play a major role in identifying and cleaving the target mRNA. RNAi functions as an important defence mechanism against parasitic nucleotide sequences, but has also an important role in the regulation of gene expression. While transient RNAi is limited to a certain time-window in the development of one generation of an insect, systemic RNAi is inherited to the next generation(s).

Self-Limiting vs Self-Sustaining

In self-limiting control strategies like the SIT, the released insects do not persist in the environment. Therefore, repeated releases are necessary to control a population. In contrast, in a self-sustaining approach, the released insects are able to persist in the wild and spread into the wild populations. Therefore, a one-time release may be sufficient, depending on several factors including the fitness and spreading capacity of the released insects, so that the replacement of the target population happens in a reasonable amount of time.

Sex Ratio Alteration

Manipulation of the natural sex ratio of $50 \%$ male and $50 \%$ female offspring in favour of one of the sexes. This can be a means to produce male-only strains of a transgenic insect, and can be achieved by manipulating the sex determination pathways in embryonic development, for example by using RNAi or by influencing sex-specific splicing.

Transgenic Insect

Relating to an insect, whose genome has been altered by the transfer of an exogenous gene. Transgenesis can occur naturally or by genetic engineering techniques. A transgenic insect created by genetic engineering falls in the category of GM insects.

Transgenic Population Suppression

Molecular technologies, that use foreign DNA transferred into an organism, resulting in the death of its progeny, fall under the term transgenic population suppression. While the effects of the SIT and transgenic population suppression are similar, i.e. population size reduction, the modes of action are different. In the SIT, population reduction is achieved through sterile matings by the released males due to irradiation-induced damaged sperm, but in transgenic approaches the population reduction is often achieved by overexpression of lethal genes at certain stages of development in the offspring of the released males (Robinson, this volume).
\end{abstract}

\title{
2. KEY MOLECULAR TECHNOLOGIES FOR POPULATION CONTROL
}

\subsection{Transposable Elements}

A frequently applied method to produce transgenic insects is using highly efficient class II transposable elements like piggyBac (Cary et al. 1989; Elick et al. 1996; Handler et al. 1998; Handler and Harrell 1999), Minos (Franz and Savakis 1991; Loukeris et al. 1995), Mariner (Mos1) (Jacobson et al. 1986; Lidholm et al. 1993) or Hermes (Warren et al. 1994; O'Brochta et al. 1996). These DNA transposons consist naturally of a transposase-encoding gene flanked by inverted repeat sequences. These elements integrate into short, defined, and randomly distributed recognition sequences in the insect genome (Handler 2002). For all transposons used commonly as molecular tools, the transposase gene has been separated from its flanking inverted repeats to create a bipartite system -- the transposase source in one vector (helper element) and a second transformation vector containing the inverted repeats flanking the transgene construct to permit its genomic integration (Handler and 
Harrell 1999, 2001a). To increase the germline transformation frequency, mutagenesis approaches have resulted in hyperactive transposases with the piggyBac variant hyPBase being highly active in diverse insects (Eckermann et al. 2018). Due to their broad applicability to many different insect species, bipartite transposable systems have become a key tool for insect transgenesis (Wimmer 2003); nevertheless, they have some drawbacks. Although the rapid loss of the nonintegrated helper element separates the integrated transgenes from a source of transposase, the possibility of unwanted transgene remobilization in the presence of an endogenous transposase remains. In addition, the essentially random integration of the transposable elements into the genome can lead to insertional mutagenesis that can be deleterious or lead to reduced fitness of the transgenic strains (Woodruff 1992; Spradling et al. 1995; Catteruccia et al. 2003; Scolari et al. 2008; Häcker et al. 2017). Moreover, the inserted transgene is subject to genomic position effects, where the presence of nearby enhancers or silencers influences transgene expression levels (Schetelig et al. 2009b). Transgenes inserted close to heterochromatic regions may also be subject to silencing due to position effect variegation, i.e. the inactivation of a gene in some cells through its abnormal juxtaposition with heterochromatin. Some of these effects can be avoided by using chromatin insulator elements (Horn and Wimmer 2003; Sarkar et al. 2006; Scolari et al. 2008) or sitespecific genome modification tools.

\subsection{Site-Specific Recombination Systems}

Site-specific recombination (SSR) systems have become important and universal molecular tools for targeted genome modification (Bode et al. 2000; Kolb 2004; Coates et al. 2005). These systems depend on a recombinase enzyme that recognizes and binds short recombination sequences, induces double-strand breaks in the central crossover region of the recombination sequence, and catalyses strand exchange. The Cre recombinase was the first enzyme to be established as a molecular tool, and it paved the way for genomic modification and conditional gene targeting in mammalian cell lines and mouse models (Sauer and Henderson 1988; Orban et al. 1992; Tsien 2016). SSR systems commonly used in mammals and insects include Cre/lox from the Escherichia coli phage P1 (Siegal and Hartl 1996, 2000), Flp/FRT from the two-micron plasmid of Saccharomyces cerevisiae (Andrews et al. 1986), as well as the phiC31/att derived from the Streptomyces phage phiC31 (Thorpe and Smith 1998). Recombination sequences of the tyrosine recombinases Cre and Flp (lox and FRT, respectively) range from 34 to 48 nucleotides, and consist of two nearly identical motifs with an inverted-repeat symmetry flanking an eight base pair (bp) central crossover sequence where the recombination takes place (Fig. 1A). Site-specific genomic integration occurs by recombination between a single sequence integrated in the genome (acceptor site) and a second sequence (donor site) in a vector containing the transgene of interest. Recombinase protomers bind to each inverted-repeat motif at both recombination sites to catalyse the strand exchange, leading to donor vector integration. The donor and acceptor sequences are identical in case of the lox and FRT sites. Thus, they remain unchanged upon completion of the recombination and are available for 
successive reactions (Fig. 1B). Site preservation, however, also has a disadvantage: donor vector integration results in duplication of the recombination sites in the genome, flanking the transgene construct (Turan et al. 2013; Häcker et al. 2017). As the reaction between cis elements is thermodynamically and kinetically favoured over the reaction in trans, the excision reaction is much faster than the integration, leading to a very low frequency of stable integration events (Baer and Bode 2001; Wimmer 2005; Häcker et al. 2017). Adding the donor plasmid in excess can help to push the reaction towards the product (Turan and Bode 2011; Turan et al. 2013).

The recombination mechanism of serine recombinases, such as the phiC31 system, follows the same basic rules as Cre and Flp. However, it strongly differs on the sequence level and the architecture of the recombination sites. The recombination sequences recognized by the phiC31 integrase are termed attP and $a t t B$, and are much longer (about $200 \mathrm{bp}$ ) than the lox or FRT sites. The two arms of the att sites flank a 3-base-pair crossover region, and in contrast to lox and FRT sites they share only limited inverted repeat symmetry (Fig. 2A). Moreover, the attP and $a t t B$ sites share no sequence identity besides the central crossover region. Therefore, recombination between $a t t P$ and $a t t B$ results in different and incompatible products, $a t t L$ and $a t t R$, making it a one-time unidirectional reaction (Fig. 2B). In principle, however, the reverse reaction should be possible in combination with additional factors/enzymes that are naturally used by the phage to excise its DNA from the host genome.

Site-specific integration into insect genomes has been established with the integrase phiC31 for several species besides drosophilids, including two Aedes species (Nimmo et al. 2006; Labbé et al. 2010; Franz et al. 2011), Anopheles gambiae Giles (Meredith et al. 2011, 2013; Isaacs et al. 2012), and the Mediterranean fruit fly (Schetelig et al. 2009b). The drawback of site-specific integration via a single recombination site is the integration of the complete donor vector at the genomic target site, including bacterial resistance genes and regulatory elements within the vector backbone that may be unnecessary or even interfere with transgene expression.

The discovery that the mutation of certain bases in the central crossover region of lox or FRT sites does not abolish cleavage by the recombinase, but renders the sites incompatible with the wild-type sites (Hoess et al. 1986; Lee and Saito 1998; Missirlis et al. 2006), opened up new possibilities (Fig. 1A). These mutant sites recombine with an identical (homospecific) mutant site with equal efficiency as two wild-type sites, while the interaction between sites with non-identical core sequences (heterospecific sites) is usually prevented. This was the basis for a more sophisticated targeting strategy, the recombinase-mediated cassette exchange (RMCE). Cre- or Flp-RMCE is based on the double-recombination between two pairs of heterospecific recombination sites, flanking DNA cassettes in the donor vector and the genomic target site (Schlake and Bode 1994; Turan and Bode 2011), which leads to a cassette exchange instead of complete donor plasmid integration (Fig. 1C). Both, Flp/FRT and Cre/lox-based RMCE, were first demonstrated in Drosophila melanogaster Meigen (Horn and Handler 2005; Oberstein et al. 2005; Wimmer 2005). 


\section{Mechanism of tyrosine recombinases}
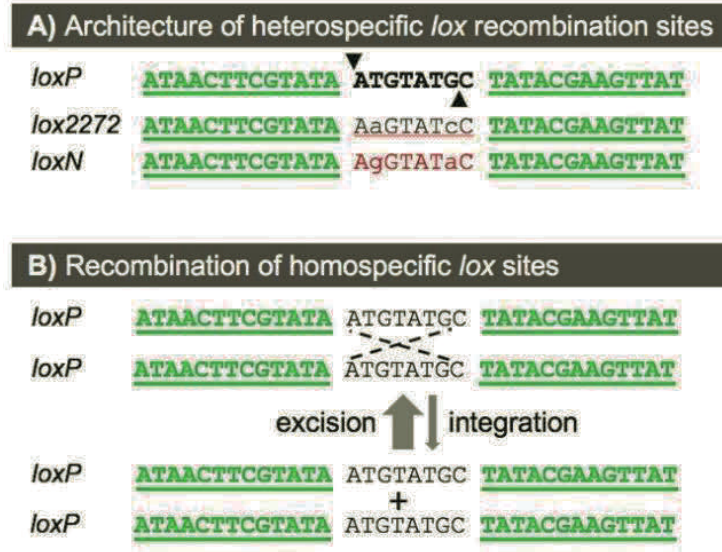

C) Mechanism of Cre-RMCE

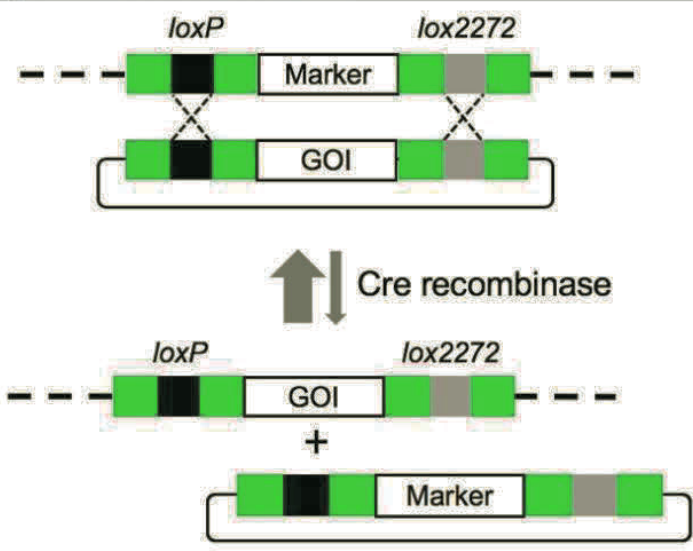

Figure 1. Comparison of site-specific recombination mechanisms catalysed by tyrosine recombinases. A) General architecture of heterospecific recombination sequences recognized by the tyrosine recombinases shown exemplarily for the Cre/lox system. Note that the FRT sites of the Flp/FRT system show the same architecture and differ only in the recognition sequence. Inverted repeat sequences flanking the 8-bp core are underlined. The heterospecific lox sites differ from the wildtype loxP site in the core sequence (mutations shown in lowercase). Only two of the existing heterospecific lox sites are shown here, B) Due to the inverted repeat architecture of the lox sites, their sequence is preserved upon recombination between identical (homospecific) sites and the reaction is reversible by the Cre recombinase. Due to the thermodynamically and kinetically favoured reaction between recombination sites in cis, the excision reaction is much faster than the integration, resulting in a very low integration efficiency. C) The genomic landing site for Cre-RMCE consists of a marker cassette flanked by heterospecific lox sites. Recombination with the corresponding homospecific sites, that flank the gene of interest (GOI) cassette on the donor plasmid, leads to a cassette exchange. Recombination sites are preserved, the reaction is reversible. 


\section{Mechanism of serine recombinases}

\section{A) Architecture of att recombination sites}

attP GGAGTAGTGCCCCAACTGGGGTAACCT TTG AGTTCTCTCAGTTGGGGGCGAGGGTC attB CCGCGGTGCGGGTGCAGGGCGTGCCC TTG GGCTCCCCGGGCGCGTACTCCACCTCA

\section{B) Recombination between att sites}

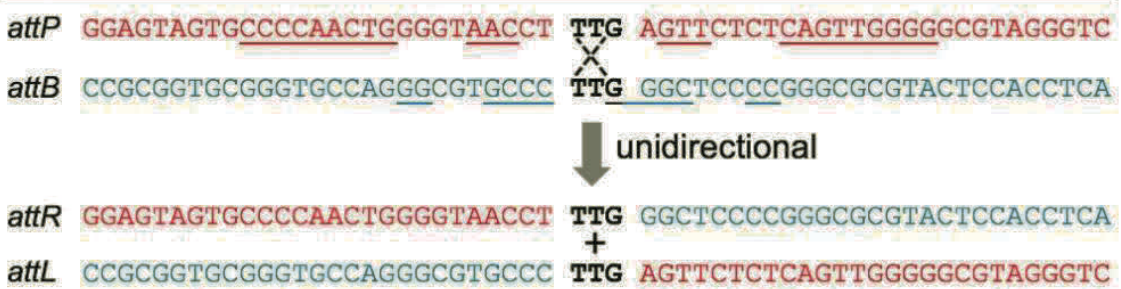

\section{C) Mechanism of $\Phi \mathrm{C} 31-\mathrm{RMCE}$}

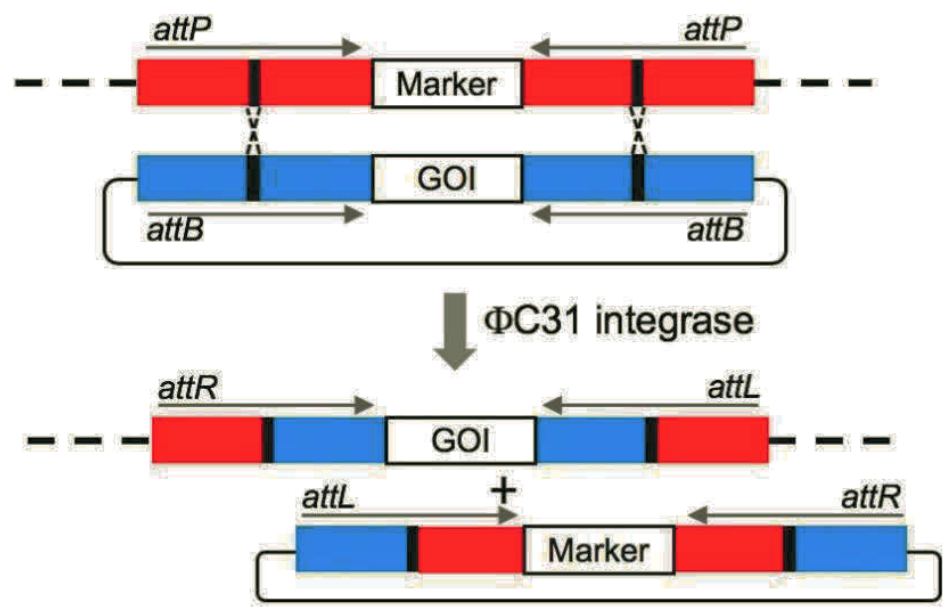

Figure 2. Comparison of site-specific recombination mechanisms catalysed by serine recombinases. A) Serine recombinase recognition sequences show very limited repeat symmetry (underlined bases). Moreover, the attP and attB recombination sites recognized by the phiC31 recombinase share almost no sequence identity besides the $3 \mathrm{bp}$ central crossover

region (TTG). B) In contrast to lox sites (and tyrosine recombinases in general), the recombination between attP and attB results in incompatible att $L$ and att $R$ sites that can' $t$ recombine anymore. The absence of the reverse reaction enhances the integration efficiency via att sites in contrast to lox of FRT sites. C) For phiC31-RMCE, the genomic marker cassette is flanked by inverted attP sites, while the donor cassette is flanked by inverted attB sites. Recombination leads to cassette exchange but is irreversible due to the resulting incompatible attR and attL sites that are not recognized by the phiC31 integrase enzyme. 
Until now, Cre/lox-RMCE was applied to only two non-drosophilid species, the tephritid Anastrepha suspensa (Loew) (Schetelig and Handler 2013a) and the mosquito Aedes aegypti (L.) (Häcker et al. 2017). Very recently, it was also achieved in the highly invasive pest Drosophila suzukii (Matsumura) (Schetelig et al. 2019). The functionality of Flp-RMCE was also demonstrated in the silkworm Bombyx mori (L.) (Long et al. 2012). In both systems, Cre- and Flp-RMCE, the heterospecific sites are restored after recombination. Therefore, both approaches allow for the repeated manipulation of the same target site including the reversion of the reaction.

The difference in the sequence of $a t t P$ and $a t t B$ sites, and the resulting directionality of the reaction, abolishes the need for heterospecific sites for RMCE using the phiC31 system. For phiC31-RMCE, the genomic acceptor cassette is flanked by attP sites in inverted orientation, while the donor cassette is flanked by inverted attB sites (Fig. 2C). This advantage, however, comes with certain shortcomings, such as the lack of control over the directionality of the cassette integration. Moreover, as for the donor plasmid integration via att-sites, the phiC31RMCE is a one-time, unidirectional reaction due to the resulting incompatible attR and attL sites. PhiC31-RMCE has been successfully demonstrated for $D$. melanogaster (Bateman et al. 2006), B. mori (Long et al. 2013), and Ae. aegypti (Haghighat-Khah et al. 2015). A different approach combines phiC31 and Flp- or Cre-recombination to achieve cassette exchange in a two-step process via a donor plasmid integration intermediate (iRMCE) (Haghighat-Khah et al. 2015).

\subsection{Genome Editing Methods}

Genome editing methods use engineered nucleases, "molecular scissors", to sitespecifically modify the genome, making use of two naturally occurring and conserved DNA repair mechanisms in the cell. The nucleases create double-strand breaks (DSBs) at desired locations in the genome. These are then repaired, either by the cell's homology directed repair (HDR) or the non-homologous end-joining (NHEJ) pathway, which results in targeted mutations. Molecular biologists have engineered four families of nucleases as genome editing tools: meganucleases, zinc finger nucleases (ZFNs), transcription activator-like effector-based nucleases (TALEN), and the clustered regularly interspaced short palindromic repeats (CRISPR)/Cas system.

Meganucleases have long recognition sequences ( $>14 \mathrm{bp}$ ) which makes them very specific. By modifying their recognition sequence through protein engineering, their rather limited choice of target sequences can be expanded (Sussman et al. 2004; Arnould et al. 2006; Rosen et al. 2006). Protein engineering, however, can be time-consuming and costly.

The concept behind ZFNs and TALENs is based on a fusion protein consisting of a non-specific DNA cutting enzyme linked to peptides that recognize specific DNA sequences, such as zinc fingers and transcription activator-like effectors (TALEs). The DNA cleavage domain used for both systems is the restriction endonuclease Fokl (Kim et al. 1996; Li et al. 2011) that is active in yeast, plant and animal cells (Wood et al. 2011). The DNA binding domain (ZNF or TALE) can be 
engineered to bind nearly any desired DNA sequence. Both methods have been applied successfully to edit a variety of insect genomes (Bozas et al. 2009; Takasu et al. 2010, 2013; Liu et al. 2012; Aryan et al. 2013; Smidler et al. 2013), and they have been shown to work with satisfying efficiency and precision. However, as for meganucleases, the number of naturally occurring target sites is limited, and the engineering of the proteins to target new sites is cumbersome and costly, preventing the widespread use of these systems.

The CRISPR/Cas9 system has recently emerged as a very powerful tool for targeted genome editing in a variety of organisms. CRISPR/Cas is the prokaryotic equivalent of acquired immunity. CRISPR are repeat-spacer sequences found to date in approximately $40 \%$ and $90 \%$ of the sequenced bacterial and archaea genomes, respectively. The short spacer DNA originates from foreign DNA, e.g. virus or plasmid, and has been acquired by the bacteria and archaea as part of the immune response to a previous infection. These spacer DNAs are separated by short repeat sequences. Small clusters of Cas (CRISPR associated) genes are located next to the repeat-spacer array (for comprehensive explanations and illustrations, see Horvath and Barrangou 2010, Marraffini and Sontheimer 2010 or Bhaya et al. 2011). These repeat-spacer arrays are transcribed and processed into short CRISPR RNAs (crRNA) by nucleases of the Cas family of proteins. The crRNAs associate with other Cas proteins into ribonucleoprotein complexes that recognize foreign DNA by sequence complementarity with the crRNA. The foreign DNA is then cut by a Cas nuclease activity in a way similar to RNA interference in eukaryotic organisms (Marraffini and Sontheimer 2010), thus conferring immunity against infection with previously encountered infective agents.

Three different CRISPR/Cas systems are known so far, consisting of Cas proteins with different functions. The most used nuclease for genome editing is the multifunctional Cas9 protein from the type II system. Cas9 requires both the crRNA and a transactivating CRISPR RNA (tracrRNA) to function. For more convenient use, scientists engineered the crRNA and tracrRNA into one single-guide RNA (sgRNA) (Jinek et al. 2012). By adjusting the sequence of the sgRNA, the CRISPR/Cas9 system can be programmed easily to target any desired genomic location that is in the vicinity of a so-called PAM (protospacer adjacent motif) sequence (Jinek et al. 2012). The system can be used to knock out existing genes or add new DNA sequences, and has been adapted to different species. One drawback of CRISPR/Cas is the potential off-target effects, requiring very careful sgRNA design. However, the system is under constant adjustment and improvement, e.g. engineering the Cas protein (Kleinstiver et al. 2016), characterizing and adapting new Cas proteins, and engineering sgRNAs (Fu et al. 2014; Nowak et al. 2016). For insects, CRISPR/Cas was first developed in D. melanogaster in 2013 (Ren et al. 2013; Yu et al. 2013; Bassett et al. 2014), and by now has been adapted for several other species including Ae. aegypti (Kistler et al. 2015), An. gambiae (Hammond et al. 2016), B. mori (Wang et al. 2013; Ma et al. 2014), Gryllus bimaculatus De Geer (Awata et al. 2015), Danaus plexippus (L.) (Markert et al. 2016), and Spodoptera litura (F.) (Bi et al. 2016), and was recently transferred to the highly invasive agricultural pests C. capitata (Meccariello et al. 2017; Aumann et. al. 2018) and D. suzukii (Li and Scott 2016; Kalajdzic and Schetelig 2017; Li and Handler 2017). 
This rapid adaptation of CRISPR/Cas to a variety of insects within just 4 years demonstrates the versatility of this method and its potential for future applications.

\subsection{Binary/Conditional Expression Systems}

Genetic sexing or sterility systems are useful only if the trait conferring sexing or sterility can be switched off for strain rearing. Therefore, binary or conditional expression systems are required. The GAL4-UAS system is a binary expression system first established in D. melanogaster (Brand and Perrimon 1993). It consists of a driver line containing the budding yeast GAL4 transcriptional activator under the control of a promoter of interest, and an effector line containing the upstream activating sequence (UAS) to which GAL4 binds to activate a gene of interest. The system becomes activated only when both parts of the system are present in an insect, i.e. by crossing the two lines. Additional control of the system is possible by the repressor GAL80 (Lee and Luo 1999) or by temperature-sensitive GAL4 versions (Mondal et al. 2007). The GAL4-UAS system has become a milestone tool for functional genomics studies in D. melanogaster, with thousands of driver and effector lines (Rorth 1998; Hayashi et al. 2002; Pfeiffer et al. 2008). The system has also been applied to several other insect species (B. mori (Imamura et al. 2003), Tribolium castaneum (Herbst) (Schinko et al. 2010), Ae. aegypti (Kokoza and Raikhel 2011), and An. gambiae (Lynd and Lycett 2012)), and could be exploited to create genetic sexing or sterility systems. However, the necessity of constantly keeping two strains to be crossed only for the release generation hinders its potential and practical application, because it increases mass-rearing complexity and costs.

The recently established bipartite Q system utilizes regulatory genes of the $q a$ gene cluster from the fungus Neurospora crassa Shear and B. O. Dodge (Potter et al. 2010), and resembles the GAL4-UAS system in that it consists of an activator QF and an upstream activating sequence QUAS. The system shows low basal expression in the absence of $\mathrm{QF}$, and high expression in the presence of QF. Expression can be efficiently suppressed by the repressor QS. The advantage of the Q system over GAL4-UAS is the additional level of control via quinic acid. Feeding quinic acid to flies temporarily relieves QS suppression, thus permitting the creation of a conditional expression system. The Q system, which was established in $D$. melanogaster and recently transferred to An. gambiae (Riabinina et al. 2016), might advance to become another conditional tool in insect genetics, biotechnology, and pest control. This would allow developing insect strains with an additional and completely independent lethality or sterility system to counter the possibility of resistance development against the first system (Eckermann et. al 2014).

Food supplement-controlled conditional expression systems like the "Tet-on" and "Tet-off" systems were developed by Bujard and Gossen (Gossen and Bujard 1992; Gossen et al. 1995) as mechanisms for inducible and reversible gene expression in eukaryotic cells. They are controlled by the antibiotic tetracycline (Tet) or one of its derivatives, e.g. doxycycline. The Tet-system is based on a naturally occurring bacterial defence system against antibiotics. It consists of the tetracycline-controlled transactivator protein (tTA) and the TetO operator sequence. tTA represents a fusion of the DNA-binding domain from the bacterial tetracycline 
repressor (TetR), and the transcriptional activation domain of the HSV1 protein VP16. For most Tet-on/Tet-off systems, several repeats of the TetO (mostly 7) are fused together with a minimal promoter, forming the Tet response element (TRE). tTA binds to the TRE and thereby triggers the expression of a downstream gene. In the Tet-off system, the binding of Tet to tTA inactivates tTA binding to TRE, therefore the downstream gene is not expressed, and the effect is "off". For Tet-on, binding of Tet to the reverse tetracycline-controlled transactivator protein (rtTA) enables binding to the TRE site and activates downstream gene expression - the system is "on". In contrast to other binary expression systems, like the GAL4-UAS system, the Tet-systems are controllable via the external addition of tetracycline. Moreover, the expression control is tighter, i.e. less leaky, than for example the estrogen receptor-based conditional expression systems (Sohal et al. 2001).

In insects, the Tet system was first tested in D. melanogaster (Bello et al. 1998), and has been applied to create conditional sterility or gender-specific lethality systems. Heinrich and Scott reported the first conditional female-specific lethality system in D. melanogaster, causing death in late pupal or early adult stages by expressing the pro-apoptotic gene head involution defective (hid) in the adult female fat body (Heinrich and Scott 2000). Such conditional lethality systems could, in general, also be constructed as a unisex lethality system that could be used in population control programmes instead of using radiation for sterilization. Since the released insects are not sterile in the strict sense, but carry a dominant lethal that would kill their offspring, Thomas et al. (2000) introduced the term RIDL "Release of Insects carrying a Dominant Lethal" as an alternative to the SIT. Gong and colleagues created a one-component Tet-suppressible RIDL system in the Mediterranean fruit fly C. capitata (Gong et al. 2005). Instead of a specific promoter driving tTA, which then activates a lethal gene, they created an autoloop where tTA activates its own expression. This leads to the accumulation of toxic levels of tTA in the late larval or pupal stages in the absence of Tet. Fu et al. (2007) then combined this system with the sex-specifically spliced Cctra-intron (section 3.1.) to conditionally kill females, and named the system female-specific RIDL (fsRIDL). The fsRIDL system was subsequently transferred to a number of insect species using either exactly the same system as in $C$. capitata or by adjusting for species-specific regulatory elements (section 3.1.).

The first conditional transgenic embryonic sexing system (TESS) based on Tetoff was developed in A. suspensa, using a combination of an early promoter, acting at the egg stage, driving the tTA expression, and hid as a lethal effector gene to cause embryo-specific lethality in the progeny. Sex-specificity is achieved again by including the Cctra-intron into the hid gene (Schetelig and Handler 2012a). Like RIDL, also the early-acting Tet-off system can be designed as a unisex lethality system based on embryonic promoters and lethal effectors (Horn and Wimmer 2003; Schetelig et al. 2009a; Schetelig and Handler 2012a, b). The use of female-specific lethality systems for sexing is described in detail in section 3.1.; for the application of unisex lethality to create sterility systems, see section 3.3. 


\subsection{RNAi}

RNA interference (RNAi) is a conserved eukaryotic mechanism of defence against viruses and foreign double-stranded RNA (dsRNA). Long dsRNA molecules are recognized and cleaved by the Dicer protein. The resulting small interfering RNA (siRNA) molecules are incorporated into the RNA induced silencing complex (RISC), and guide it to the complementary target messenger RNA (mRNA) that is cleaved by RISC and thereby silenced. RNAi has become an important tool to study gene functions, also in insects (Bucher et al. 2002). Many studies were performed by microinjection of dsRNA into eggs, larvae or adults, but this procedure is not applicable for mass-producing insects. Therefore, the finding that oral ingestion of dsRNA can trigger RNAi opened the door for its use in pest control. Oral delivery of dsRNA in insects was first described in the triatomine bug Rhodnius prolixus Stål and the light brown apple moth Epiphyas postvittana (Walker) (Araujo et al. 2006; Turner et al. 2006).

The first topical application of dsRNA that also induced systemic RNAi (when the signal spreads throughout the insect's body) was shown in the Asian corn borer Ostrinia furnacalis (Guenée) (Wang et al. 2011). Systemic RNAi by topical application of IAP1 dsRNA in Ae. aegypti could not be confirmed despite a first positive report (Pridgeon et al. 2008, 2016). In contrast, it was shown that topical application of the Aae IAP1 dsRNA did not to have a significant knock-down effect on the mRNA level, and even microinjection of IAP1 and other IAPs into adult $A e$. aegypti females did not cause significant mortality (Puglise et al. 2016). Therefore, it cannot be determined if failure of the topical application in Ae. aegypti was due to the method or the target selection.

Both methods, oral ingestion and topical application, require in vitro RNA synthesis, which on a large scale can become costly. Another option is the in vivo delivery via bacteria (Tian et al. 2009; Zhu et al. 2011), host plants (Baum et al. 2007; Mao et al. 2007; Pitino et al. 2011; Zha et al. 2011), or host-plant viruses (Kumar et al. 2012) expressing the dsRNA. All three strategies have been shown to induce systemic RNAi in the investigated species: western corn rootworm Diabrotica virgifera virgifera LeConte (Baum et al. 2007), cotton bollworm Helicoverpa armigera (Hübner) (Mao et al. 2007), tobacco hornworm Manduca sexta (L.) (Kumar et al. 2012), brown planthopper Nilaparvata lugens Stål (Zha et al. 2011), and green peach aphid Myzus persicae (Sulzer) (Pitino et al. 2011). The bacteria-based system had a stronger effect than delivering the dsRNA via host plants. A possible reason for the reduced effect via host plant delivery might be the processing of the produced dsRNA by the plant's own RNAi machinery and Dicer enzymes into siRNA molecules, which might be less effective in the insect than the dsRNA (Mao et al. 2007; Kumar et al. 2012). This hypothesis is in line with an observation in the western corn rootworm where dsRNAs (but not siRNAs) are internalized into the midgut tissue (Ivashuta et al. 2015).

Given careful target design (which includes target selection and design of dsRNA taking into account polymorphisms and paralogous genes), RNAi can be a very specific approach. This has been demonstrated by Whyard et al. (2009) who showed that, by targeting the same enzyme in four different species, one can selectively kill only the species for which the dsRNA was designed. However, 
biological barriers, and not the proper target gene selection, are the most important limiting factors in insect RNAi. First, an efficient uptake by the midgut epithelial cells is required. Second, the alkaline environment in the insect midgut, as in lepidopterans (Dow 1992), could present a problem due to lysis of the dsRNA. Third, another significant barrier is the presence of nucleases in the midgut and salivary glands of insects. These nucleases might also be the reason for the lack of systemic RNAi in some insects. This is supported by a comparative study which showed that, in the RNAi-insensitive lepidopteran $M$. sexta, the dsRNA is rapidly degraded in the hemolymph, but in the hemolymph of the RNAi-sensitive cockroach Blattella germanica (L.) the dsRNA is stable (Garbutt et al. 2013). Along the same line, the desert locust Schistocerca gregaria (Forskal) shows a potent systemic RNAi response to injected dsRNA, but is insensitive to ingested dsRNA (Wynant et al. 2012). This phenomenon was explained when four different midgut RNAses were identified in S. gregaria (Wynant et al. 2014). Nevertheless, it might still be possible to target insects not susceptible to systemic RNAi by targeting midgutspecific genes, e.g. genes involved in detoxification pathways that are accessible for ingested dsRNA, provided that there are no midgut RNAses present.

In contrast to transgenic insects, the effect of RNAi is mostly transient (except for some cases of systemic or transgenerational RNAi). Therefore, the dsRNA must be supplied constantly during relevant developmental stages to guarantee the silencing effect. Depending on the production costs for the dsRNA and the required amounts (which depends on the production scale of the insects and the effectiveness of the dsRNA to silence the target gene), RNAi might be a costly method for pest control. An alternative to the synthetic production of dsRNA is the use of dsRNAproducing transgenic plants for agricultural pests or feeding the insects during massrearing with bacteria expressing the dsRNA. While this decreases the costs of dsRNA production, it involves using transgenic organisms, and with them come regulatory constraints in most countries, thus eliminating the advantage of RNAi (which by itself is a non-transgenic approach). Moreover, as the bacterial strain commonly used to express the dsRNA does not colonize the insect gut, this delivery method also has only transient effects. Recently, Whitten et al. (2016) succeeded in modifying symbiotic gut bacteria to express the dsRNA; these bacteria recolonized the gut of the target species and thereby induced a long-term silencing effect. Another option to circumvent the only transient effect of RNAi is by using transgenic insects expressing the dsRNA themselves. However, this would require again a conditional expression system (section 2.4.) to suppress dsRNA production for rearing the strains.

Several comprehensive reviews summarize the current insights and achievements in insect RNAi, and describe the challenges that are faced when developing this method as a tool for use in pest control (Baum and Roberts 2014; Joga et al. 2016; Darrington et al. 2017; Cooper et al. 2019). 


\section{APPLYING MOLECULAR TECHNOLOGIES TO IMPROVE THE SIT}

Modern molecular technologies can be used potentially to address and improve key aspects of programmes integrating the SIT such as sexing, sterilization, and marking of the insects destined for release. Combining available knowledge on insect biology, e.g. sex determination pathways, with specific and targeted genome modifications, could pave the way for new pest control systems. The following sections describe the current knowledge on sexing, sex determination and distortion, sterilization, monitoring, and transgene stabilization, as well as existing genetic (transgenic) systems with potential application for the SIT.

\subsection{Sexing}

The release of only male insects can enhance the efficacy of SIT applications because the presence of sterilized females appears to prevent some sterilized males from competing with wild males for matings with wild females. Large-scale field studies comparing mixed sex versus male-only releases of the Mediterranean fruit fly in Guatemala showed a three- to five-fold increase in efficacy (Rendón et al. 2004). Moreover, male-only releases are a prerequisite in the case of vector control programmes because, for example, released female mosquitoes (even if sterile) would increase the number of biting and potentially disease-transmitting insects. For agricultural control programmes, it may also be beneficial not to release females, because the sterilized females could still cause damage by laying their eggs into fruits. Another important factor of male-only rearing is the reduction in costs, space, and logistics required at all levels of pest control programmes that apply the SIT, i.e. mass-rearing, quality control, distribution, and release as well as monitoring (Cáceres et al. 2004).

Significant savings can be gained if females are eliminated early in the rearing process (Franz et al., this volume). The role model for sexing is the Mediterranean fruit fly temperature-sensitive lethal ( $t s l$ ) strain developed by classical genetic approaches, where the $t s l$ mutation specifically and effectively kills all female embryos upon heat shock (Franz and McInnis 1995; Franz et al., this volume). Recently, another $t s l$ mutation was created successfully by chemical mutagenesis in the mosquito An. arabiensis (Ndo et al. 2018). However, at the present time, the $t s l$ mutations are not identified molecularly, and the creation of such a mutation using classical mutagenesis often takes a long time. Nevertheless, once the molecular basis of such a mutation is identified, modern molecular tools, as described above, could be used to build this highly efficient system in other species to create new conditional sexing strains based on heat shock, thus avoiding chemicals and antibiotics. These new strains might be classified as non-transgenic since no foreign DNA is transferred to the insect of interest, and potentially even as non-GM since the modification is based on a naturally occurring mutation. Thereby these sexing strains would evade the regulatory restrictions that apply to the release of GM organisms.

Other strategies could rely on the use of sex-determining factors to produce male-only strains. Sex-determination pathways in insects are very diverse, but all 
appear to be centred around a highly conserved sex-determining factor in Diptera, Lepidoptera, and Hymenoptera, doublesex ( $d s x)$, at the end of a sex-determination cascade (Funaguma et al. 2005; Verhulst et al. 2010; Geuverink and Beukeboom 2014). Dsx is sex-specifically spliced into a male and female isoform. In many tephritids this is controlled by transformer (tra) (Saccone et al. 2011) as a master epigenetic switch. A functional tra protein seems to be produced only in females $(\mathrm{XX})$, while the male pre-mRNA is not spliced, and therefore contains an early stop codon leading to a non-functional protein (Verhulst et al. 2010). The female tra protein functions as splicing enhancer, leading to the female-specific splicing of $d s x$. In contrast, the absence of functional tra protein in males results in male-specific splicing of $d s x$. Female-specific tra expression is maintained through a positive feedback loop. When zygotic activation of this loop is prevented, male development follows. Although the function of tra appears to be conserved across many species, its sequence is not (Pane et al. 2005; Verhulst et al. 2010). This not only makes the identification of species homologues difficult, but also allows diversity of upstreamacting factors in different species. Therefore, deciphering the sex-determination pathways and other factors involved in different insects can be challenging.

Recently, breakthroughs were achieved in several insect species. Scientists identified the long-sought $\mathrm{M}$-factor Nix in Ae. aegypti and Ae. albopictus (Hall et al. 2014, 2015; Liu et al. 2020), the maleness gene Yob in An. gambiae (Krzywinska et al. 2016), GUY1 in Anopheles stephensi Liston (Criscione et al. 2013, 2016), Mdmd in Musca domestica L. (Sharma et al. 2017), and the Maleness-on-the-Y (MoY) gene in C. capitata (Meccariello et. al. 2019). Nix, Yob, GUY1, and Mdmd are located upstream of $d s x$ in the cascade, and are needed for male development by directly or indirectly inducing male-specific $d s x$ splicing. In the Mediterranean fruit fly, where tra is maternally deposited in the embryo to induce its auto-regulatory femalespecific splicing, $M o Y$ is necessary and sufficient to suppress this female-specific Cc-tra splicing, resulting in male development. However, the mechanism of suppression has not yet been solved. Interestingly, $M o Y$ is functionally conserved in two other tephritids, Bactrocera oleae (Rossi) and B. dorsalis, providing a candidate gene for genetic-control strategies. Another mechanism of female-specific splicing of $d s x$ was discovered in the WZ sex-determination system of the lepidopteran $B$. mori, where it is induced by a female-specific piRNA (piwi-interacting RNA) (Kiuchi et al. 2014).

The sex-determining factors could be promising tools to eliminate females or convert them to males for SIT projects (Schliekelman et al. 2005). The sexspecifically spliced $C$. capitata tra-intron is already successfully being used to robustly eliminate females. The female-specific splicing of the tra-intron leads to a functional protein, whereas the male isoforms result in truncated, non-functional versions. This has been exploited to develop a Mediterranean fruit fly sexing system using the Cctra-intron to female-specifically express a toxic gene in the fsRIDL system (Fu et al. 2007), which was also directly applied to the olive fruit fly B. oleae (Ant et al. 2012). Recently, the system was adapted to the Australian sheep blow fly Lucilia cuprina (Wiedemann) and the New World screwworm Cochliomyia hominivorax (Coquerel) using the sex-specific first intron of the screwworm tra gene for both species to female-specifically overexpress tTA to toxic levels ( $\mathrm{Li}$ et al. 
2014; Concha et al. 2016). The successful characterization of the lepidopteran doublesex $(d s x)$ gene was the basis for transferring the fsRIDL to the pink bollworm Pectinophora gossypiella (Saunders) and the diamondback moth Plutella xylostella (L.) using sex-specific splicing of $d s x$ to accumulate tTA in females (Jin et al. 2013). fsRIDL kills females in the late larval or pupal stage (Fu et al. 2007). In contrast, in the TESS, the early embryonic and female-specific expression of the pro-apoptotic gene hid using the sry-alpha promoter kills females early in embryonic development in A. suspensa, A. ludens, and C. capitata (Schetelig and Handler 2012a; Ogaugwu et al. 2013; Schetelig et al. 2016), which in a large-scale production would reduce the cost of rearing. Similar systems have recently been developed for Lucilia cuprina (Yan and Scott 2015) and C. hominivorax (Concha et al. 2016; Scott et al. 2017). Following this approach, the An. gambiae Yob might be a promising new candidate gene to create a mosquito sexing strain, because overexpression results in female lethality, potentially due to a role in dosage compensation (Krzywinska et al. 2016; Krzywinska and Krzywinski 2018).

Instead of eliminating females, male-only populations could be created by reversing females into males. This would have the advantage of doubling the male output. Sex reversion via a transient RNAi effect has been successfully performed in several species by targeting transformer genes, whose function is required only for female development. In A. suspensa, injection of dsRNA against Astra and Astra-2 into fertilized embryos led to at least $97 \%$ male progeny, of which half were XX males. These XX phenotypic males mate successfully but are infertile due to hypertrophic testes and reduced sperm motility (Schetelig et al. 2012). In contrast, transient RNAi against tra in C. capitata, B. oleae, and L. cuprina (Pane et al. 2002; Lagos et al. 2007; Concha and Scott 2009) resulted in fully transformed XX males, which were also fertile. Sex conversion could also be achieved by overexpressing suitable sex-determination genes or maleness factors, like Nix in Ae. aegypti (Hall et al. 2015; Aryan et al. 2019).

A different approach to sex distortion is the so-called $X$ shredder. The endonuclease I-PpoI specifically cuts X-chromosomal ribosomal rDNA sequences in An. gambiae. Tissue-specific expression of I-PpoI in the testes using beta2tubulin ( $\beta 2-t u b)$ 5' and 3' regulatory sequences leads to shredding of $\mathrm{X}$ chromosomes during gametogenesis, resulting in a strong bias for $\mathrm{Y}$ gametes, but also male sterility in crosses of heterozygous males with wild-type females. This is due to the long half-life of the I-PpoI protein, which is transferred via sperm into the zygote where it shreds the $\mathrm{X}$ chromosome of the oocyte upon fertilization (Windbichler et al. 2008). The system was optimized to create a pure sex-distortion system without male sterility by reducing the half-life of the I-PpoI protein through protein engineering (Galizi et al. 2014). Recently, the same group successfully rebuilt the X shredder in An. gambiae based on CRISPR/Cas. The guideRNAs were designed to recognize a conserved rDNA target sequence of the An. gambiae complex. By expressing the Cas9 protein specifically during spermatogenesis, the $\mathrm{X}$ chromosome in sperm is shredded. The selected guideRNA target sequence is conserved among An. arabiensis, An. gambiae, An. bwambae White, An. melas Theobald, and An. merus Dönitz. Therefore, this CRISPR/Cas-based X shredder should be applicable to the complete An. gambiae complex (Galizi et al. 2016). 


\subsection{Marking/Monitoring/Sex Sorting}

To be able to monitor the effect of SIT releases, sterile insects have to be distinguished from wild insects in the field (Dowell et al., this volume; Franz et al., this volume; Parker, Mamai et al., this volume; Vreysen, this volume). Traditionally, colourful adhesive powders are used to mark the sterile insects before release (Schroeder and Mitchell 1981). However, the colourful powders are error-prone in some species because of a possible transfer of the marker during contact with wild insects, or removal of the powder by grooming. Moreover, some of the dusts can be harmful to workers handling the dye. An alternative would be replacement with genetic markers coding for fluorescent proteins stably integrated into the genome of insects (Hagler and Jackson 2001; Hendrichs and Robinson, this volume). Such genetic fluorescent markers are available in a variety of colours, and can be selectively expressed by using specific promoters. Transgenic strains carrying fluorescent markers have been developed in a wide variety of insect pests, including the mosquitoes Ae. aegypti and An. gambiae (Catteruccia et al. 2005; Smith et al. 2007) as well as fruit flies like A. suspensa and C. capitata (Handler and Harrell 2001a, b; Scolari et al. 2008; Zimowska et al. 2009). Important for the use of fluorescent markers in field monitoring is the stability of the fluorescent proteins over a long time under trapping conditions. This has been shown for the pink bollworm, where DsRed proteins were stable for at least 2 wk after trapping of specimens as confirmed by fluorescence microscopy and PCR (Simmons et al. 2011). A similar study with a transgenic strain of $A$. suspensa proved the stability of DsRed fluorescent marker protein over a period of $3 \mathrm{wk}$ in field traps, monitored by visual inspection and PCR (Nirmala et al. 2011). Studies in A. ludens confirmed the marker stability under dry conditions over several months (Meza et al. 2011). As the fluorescent protein degrades over time, PCR assays have been developed to verify the DsRed marker molecularly (Zimowska et al. 2009).

Besides monitoring released insects in the field, fluorescent markers could serve multiple purposes. By using the $\beta 2$-tub promoter, the marker is expressed in the male sperm (Catteruccia et al. 2005; Scolari et al. 2008). Thus, via marker expression in the gonads, such strains can be used to monitor the mating success of the released males by screening for the fluorescent sperm in the spermathecae of wild females (Juan-Blasco et al. 2013). Moreover, the male- (or sex-) specific expression of fluorescent markers allows (automated) sexing, as shown for Aedes and Anopheles species. A transgenic Ae. aegypti strain has been constructed in which DsRed expression is driven by the $\beta 2$-tub promoter, allowing reliable sorting of DsRed males from non-marked females using the Complex Object Parametric Analyser and Sorter (COPAS) (Smith et al. 2007). In a transgenic strain of $A n$. stephensi, $\beta 2-t u b$ drives the expression of an enhanced green fluorescent protein (EGFP) permitting late larval automated sorting (Catteruccia et al. 2005). Marois and colleagues used the $d s x$ promoter to drive EGFP in An. gambiae, which permits early larval separation by COPAS (due to the higher expression of EGFP in the midgut in male L1 larvae compared with females) (Marois et al. 2012). Importantly, the COPAS sorting does not significantly affect the viability and competitiveness of sorted males (Catteruccia et al. 2005; Smith et al. 2007). 
Instead of using suitable promoters to express the markers sex-specifically, transgenic lines with insertions of the marker on the Y chromosome can be used, as available for A. suspensa: four Y-linked Dmel-PUb-DsRed lines have been created, of which one expresses the marker already in early embryogenesis (Schetelig and Handler 2013b). Ideally, a Y-chromosome insertion should also contain a landing site, e.g. attP, lox, or FRT, for site-specific integration, such that any construct of interest can be inserted into the $\mathrm{Y}$ chromosome. Such lines are available for $A n$. gambiae (Bernardini et al. 2014) and A. suspensa (Schetelig and Handler 2013b) having a $\mathrm{Y}$-linked $a t t P$ site.

\subsection{Sterilization}

Genetic sterilization approaches can also contribute to the population control of insect pests and disease vectors. One of the first genetic sterilization systems causes reproductive sterility by the transfer of embryonically lethal transgenes. It was developed in D. melanogaster (Horn and Wimmer 2003), and was later successfully adapted and transferred to important pest insects like C. capitata, Anastrepha ludens, and A. suspensa (Schetelig et al. 2009a, 2016; Schetelig and Handler 2012b). It is based on the conditional expression of a lethal effector molecule, hid, controlled by an early embryonic promoter, to cause embryo-specific lethality in the progeny. During mass-rearing the conditional systems can be switched off by a food supplement, or the antibiotics tetracycline or doxycycline. Such strains, if released into the field where no antibiotics are present, will lead to biologically fertile matings, but their progeny will die due to the embryonically active lethal system. This results in the reduction of the wild-type population.

In contrast, RIDL was designed as a late-acting conditional lethality system that kills progeny in late larval or pupal stages by accumulation of toxic levels of tTA. RIDL systems that confer unisex lethality in the next generation, and can be used as sterility systems, were designed for C. capitata and Ae. aegypti (Gong et al. 2005; Phuc et al. 2007; Harris et al. 2011). More details about the design and function of the RIDL and conditional expression systems (Tet-on/Tet-off) and their applications are given in section 2.4 .

\subsection{Transgene Stabilization}

Most transgenic insects have been routinely generated using germline transformation procedures and transposable elements as tools to randomly integrate DNA into genomes. To prevent instability or a horizontal gene transfer through the transposon system to other insect species, all commonly used transposons have been split into two non-autonomous parts, one vector encoding the transposase gene (helper), and a second vector containing the inverted repeats flanking the transgene construct to be integrated into the genome. After injection into the germline, the transposase is only transiently expressed from the plasmid that quickly gets lost during cell divisions, while the transgene should be stably integrated into the genome. However, as long as the intact inverted terminal repeats (ITRs) of the transposons flank the transgene 
construct in the genome, it can be remobilized in the presence of a suitable transposase enzyme, which could lead to a loss of function. Thus, endogenous, related cross-mobilizing transposases, could potentially remobilize the construct.

An additional concern has to do with the risk of horizontal gene transfer through the uptake of foreign DNA by other organisms in the field and the subsequent remobilization of transposons contained therein by various means. Although this risk is minimal, the possibility exists. For the use of transgenic insects in SIT projects, this possibility could be investigated by feeding the transgenic organism to other organisms in question and assess potential horizontal transfer events by molecular techniques.

Regarding remobilization, such events could be prevented by removing one or both inverted repeats. Mechanisms described for D. melanogaster and C. capitata introduce additional ITRs such that additional ITR-flanked cassettes are created at the genomic integration site. A subsequent remobilization experiment either remobilizes the two outermost ITRs, which removes the whole transgene construct (the unwanted side product), or one of the outer ITRs remobilizes with the internal ITR, thus leaving the organism with the third ITR and part of the transgene construct (the desired product). Studies verified that one ITR is not sufficient anymore to remobilize the transgene, which is therefore considered as "stabilized" (Handler 2004; Dafa'alla et al. 2006; Schetelig et al. 2009b; Long et al. 2015).

\subsection{Quality Control of Transgenic Lines}

The success of large-scale operational programmes with an SIT component depends on the regular monitoring and evaluation of the quality of the strain used (Franz et al., this volume; Parker, Vreysen et al., this volume; Vreysen, this volume). After several decades of successful development and implementation of the SIT, the FAO, IAEA, and USDA have developed standard operating procedures for the evaluation of the biological quality of strains used in mass-rearing and SIT applications (FAO/IAEA/USDA 2019). The most important parameters are rearing efficiency and sterile male performance, including mating competitiveness.

The VIENNA 8 strain is the non-transgenic genetic sexing strain, produced through classical genetics, currently used in large-scale operational Mediterranean fruit fly SIT programmes worldwide (Augustinos et al. 2017; Franz et al., this volume). VIENNA 8 exists in two versions, with and without the inversion D53, which is used to suppress recombination and ensure the genetic stability of the strain under large scale production: VIENNA $8^{\text {D53+ }}$ and VIENNA $8^{\text {D53- }}$ (Augustinos et al. 2017). Its sexing properties are based on two selectable markers, the white pupae $(w p)$ and the temperature-sensitive lethal (tsl) genes. A transgenic version of this strain was developed, VIENNA 8-1260, which carries two fluorescent markers, the DsRed in the body and the GFP in the testes (Scolari et al. 2008). In a recent study, the Mediterranean fruit fly VIENNA $8^{\mathrm{D} 53+}$ strain was compared with the transgenic strain VIENNA 8-1260 with respect to the parameters egg production, egg hatch, and egg sterility under semi-mass-rearing conditions, and male mating competitiveness in field cages (Rempoulakis et al. 2016). VIENNA 8-1260 produced significantly fewer eggs as compared with the classical genetic sexing 
VIENNA $8^{\text {D53+ }}$ strain, while egg hatch and egg sterility were similar. However, after ten generations under continuous semi-mass-rearing conditions, the male mating competitiveness of the VIENNA 8-1260 strain, when compared with wild-type males, was statistically significantly lower than that of the classical genetic sexing VIENNA 8 strain (Rempoulakis et al. 2016). The VIENNA $8^{\text {D53+ }}$, VIENNA $8^{\text {D53-, }}$ and VIENNA 8-1260 strains were also tested on a mass-rearing scale. The analysis showed that the transgenic VIENNA 8-1260 strain had significantly lower yield than both VIENNA $8^{\text {D53+ }}$ and VIENNA $8^{\text {D53- }}$ strains. In addition, the relative sterility index and male mating performance of the VIENNA 8-1260 was significantly lower

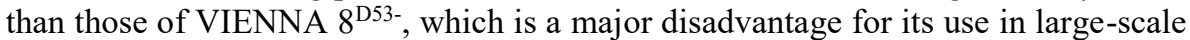
operational SIT programmes (Ramírez-Santos et al. 2017a). Another limitation of this transgenic strain is that it requires higher irradiation doses to eliminate the possibility of the vertical transfer of the fluorescent gene into wild flies and the environment (Ramírez-Santos et al. 2017b). However, the required higher irradiation doses compared with the classical genetic sexing strain VIENNA 8 may further reduce the mating competitiveness of the transgenic strain VIENNA 8-1260 (Ramírez-Santos et al. 2017b; Bakri et al., this volume).

Until now, only a few transgenic strains (and of only two species, C. capitata and $A$ e. aegypti) have been evaluated with regard to their potential suitability for use in large-scale open-field releases. In a recent study, a $C$. capitata fsRIDL strain, OX3864A, was assessed under field-cage conditions in comparison with the VIENNA $8^{\text {D53+ }}$ strain and wild-type males for its male sexual competitiveness (Virginio et al. 2017). For both strains, the mean duration of mating events was significantly lower than for the wild type. Moreover, the latency period, i.e. the time elapsed until mating starts, for transgenic males was significantly longer than for VIENNA $8^{\mathrm{D} 53+}$. In contrast, there were no differences observed in the proportion of females mated, and in the relative sterility index.

Transgenic RIDL strains of Ae. aegypti have been developed and tested for their rearing efficiency and male mating competitiveness. A female-specific RIDL version of an Ae. aegypti strain (fsRIDL) was tested in outdoor field cages; it failed to eliminate a target population because of its low mating competitiveness (Facchinelli et al. 2013). The bisexual Ae. aegypti OX513A RIDL strain has, in several places, successfully suppressed the population of this major mosquito vector species (see below). It is the only transgenic mosquito line which has been semimass-reared for releases of up to 1-1.5 million male mosquitoes per week (Carvalho et al. 2014). However, in addition to its low male mating competitiveness (thus releases were done with very high transgenic to wild male ratios), this RIDL strain also presents additional disadvantages. It is not a sexing strain, meaning that sex separation based on pupal size is very labour intensive and inefficient, thus some fertile females are released into nature; this raises concerns about their role in disease transmission as well as in spreading the transgene and leaving OX513A's genetic footprint in the environment (Bourtzis et al. 2016). Moreover, a recent study showed introgression of OX513A's genetic background into the local population, resulting in hybrids of originally genetically quite distinct populations with potentially increased robustness, and an unclear vectorial capacity (Evans et al. 2019). The rearing of this transgenic Ae. aegypti OX513A RIDL strain also depends 
on the use of tetracycline in mass-rearing. The use of this antibiotic may have a negative impact on the mosquitoes' endogenous microbiota -- known to be important in the biology, physiology, and ecology of the host, potentially affecting the rearing efficiency and mating competitiveness of the strain (Bourtzis et al. 2016).

During the past 15 years, numerous so-called landing-site lines for site-specific genome modification have been created in many insects. These lines carry transgene constructs with recombination sites for site-specific recombination systems like Cre/lox, Flp/FRT or PhiC31/att (section 2.2.), and could be used to build transgenic strains for population control programmes. Since all of these lines were created by transposon-mediated transformation resulting in the random transgene integration into the genome, the integration site could have a negative effect on the biological quality of these lines, and thereby on their suitability for large-scale pest control programmes. Therefore, it is crucial to assess the fitness of such lines.

Such assessments have been performed recently for several landing-site lines of the vector Ae. aegypti in a comprehensive set of laboratory-scale tests (Häcker et al. 2017). The lines carry a construct for site-specific genome modification via CreRMCE. The tests included parameters important for successful and economical mass-rearing, e.g. female fecundity and fertility, and time for larval development. In addition, they included parameters important for releases such as male mating competitiveness and adult male longevity. They were based on a statistical sample size estimation to ensure the detection of differences in quality of up to five percentage points compared with the parental strain Higgs White Eye (HWE). The tests showed that one of the lines, AH0212-F1, with an intergenic integration site of the transgene construct, performed closest to the parental HWE, and thus is the most suitable line for downstream site-specific modifications.

However, the tests also showed that transgene integration at sites annotated as intergenic (DNA sequences located between genes) does not necessarily indicate a "good" integration site regarding fitness costs, as observed for the line AH0212-M1 (Häcker et al. 2017). This line showed strong deficits in several parameters including female fecundity and longevity, and pupation time. This effect is purely due to the integration site since the transgene construct is identical in all lines. None of the lines, however, performed "good" or "bad" in all of the quality parameters, because different integration sites will influence different sets of genes or geneexpression pathways, thus influencing various life traits. Moreover, experiments analysing the larval hatch rate under different food conditions (baker's yeast as minimal Ae. aegypti food source in the hatching water versus TabiMin fish food as optimal food source) revealed a strong influence of the chosen experimental conditions on the results of the fitness tests. When using an optimal food source, no significant differences in larval hatch rates between the transgenic lines and the HWE reference strain were observed. However, significantly decreased hatch rates for some lines were revealed when the lines were put under "stress" by hatching them using minimal food (Häcker et al. 2017). All of the above-mentioned assessments were performed under small-scale laboratory conditions. The next step is to evaluate these lines under semi-mass-rearing conditions, and finally the best line will be assessed in a large-scale mass-rearing setting. 


\section{SUCCESSFULLY APPLYING MOLECULAR TOOLS - FIRST RELEASES OF TRANSGENIC INSECTS}

The first open-field tests with transgenic insects were performed in 2007/2008 with the pink bollworm strain OX1138B; this strain carried a transgenic marker for field monitoring. After the first field trials in 2007, when 1.1 million moths were released in Yuma County, Arizona, in 2008 this was increased to over 15 million moths released within the framework of an operational demonstration trial. The performance of the moths was equal to that of the traditional non-transgenic APHIS strain established for the ongoing SIT programme against the pink bollworm, i.e. in terms of mating success, dispersal, and persistence in the release area (Simmons et al. 2011).

The first open-field trials with transgenic Ae. aegypti mosquitoes were started in 2009 and 2010 in Grand Cayman (Harris et al. 2011), followed in 2010 by a larger scale release; over a 23-week period, 3.3 million engineered OX513A males were released in three adjacent areas. This trial showed the potential of the transgenic control strategies for mosquitoes in general, and of the OX513A in particular, as it achieved 80\% population suppression at an overflooding ratio of 5:1 (Harris et al. 2012). If there is a link between the disease-carrying insects and the epidemiological burden (which should be further evaluated in the future), this reduction in the insect population is most important and valuable. Subsequent to the success of the Grand Cayman study with OX513A, a sustained release of this strain, for a period of more than one year, was done in 2015 in a suburb of Juazeiro, Bahia, Brazil, reducing the local population by $81 \%$ (Carvalho et al. 2015). The most recently published study on OX513A additionally addresses the important question of recurrence of mosquito populations following post-release interruption in two distinct release areas in Brazil (Garziera et al. 2017). The study showed that mosquito populations recovered within 17-32 weeks after the end of the releases, demonstrating the necessity of continuous releases for permanent population suppression. Field trials with OX513A are also scheduled for the Florida Keys after the 2016 final decision by the FDA that no significant impacts on the environment are expected, although these have until now not been initiated in view of public opposition.

Field trials with Wolbachia-infected Ae. aegypti have been successfully performed in Australia, starting in 2011, with a self-sustaining (population replacement) objective (Hoffmann et al. 2011). More releases of Wolbachia-infected Aedes males are ongoing or planned in Brazil, China, Singapore, and the USA, although mainly as part of self-limiting (IIT) approaches (Callaway 2016; Waltz 2017).

A large-scale performance evaluation is also planned for a transgenic sexing strain of the New World screwworm; it will be tested for its performance in massrearing by the Comisión Panamá - Estados Unidos para la Erradicación y Prevención del Gusano Barrenador del Ganado (COPEG). An application to conduct open-field trials has been submitted to the government of Panama (Concha et al. 2016). 


\section{CONCLUSIONS AND FUTURE CONSIDERATIONS}

Modern molecular technologies offer many possibilities to address issues and bottlenecks in current insect pest and disease-vector population suppression programmes, e.g. the sexing of insects for male-only releases; for many species this is a critical tool in establishing an efficient large-scale operational programme. In addition, sterilization and marking techniques can be developed and combined to create sterile-male insect strains of good biological quality for population control. These modern biotechnologies permit strains to be tailored to meet the requirements of control programmes, and have the potential to facilitate greatly the transfer of successful strategies to new pest species in just a few years. However, for a species to be effectively targeted with these technologies, the availability of genome sequence data is beneficial, and molecular tools for insect transformation have to be available in the species of interest. For the Mediterranean fruit fly, such a resource has been built up (Papanicolaou et al. 2016), and in combination with a strong understanding of the chromosomal organization resulting from research and development of the SIT for this species, this database and knowledge will help to further identify crucial markers, genes, and targets for related tephritid and other pest species. However, high-quality genomes with mostly manual annotations are still limited, and, although the i5k insect genome project (Robinson et al. 2011) is sequencing multiple insect species, it will take many years before high-quality genomes will be available.

The CRISPR/Cas technology will also benefit from available sequence information, and promises to be a key method for editing genomes in the coming years. Current drawbacks of the method, because of off-target effects, are continuously being addressed, and the system is being improved and refined. CRISPR/Cas is only one of the techniques that can be used for creating strains useful for the suppression of populations of insect pests and disease vectors. There are several other techniques available, with distinct advantages, which can complement those of CRISPR/Cas. Thus, while CRISPR/Cas is highly flexible regarding the choice of the target site, Cre- or FLP-RMCE offer the possibility of successively targeting the same site, permitting the modification and adjustment of existing systems to changing requirements.

Until now, the described population suppression strategies for SIT applications are based on self-limiting genetic systems; they are suitable for both disease vectors and agricultural pests. In contrast, population replacement strategies make most sense in species that are transmitting a disease but are not pests by themselves, e.g. mosquitoes or vectors of plant diseases. In such insects, replacing the natural population with insects refractory to infection with the pathogen could be a viable option for disease control. However, such approaches have the disadvantage that they leave an "ecological footprint", and unexpected complications would have to be controlled by yet another self-sustaining genetic system controlling the problematic first one.

Research on such refractory strains has been performed mostly with regard to dengue and Zika infections in Ae. aegypti, using a Wolbachia-based replacement approach (Moreira et al. 2009; Hoffmann et al. 2011; Walker et al. 2011; Dutra et al. 2016; Lees et al., this volume). Moreover, trypanosome refractoriness in Glossina 
morsitans morsitans Westwood was achieved using a paratransgenesis approach (De Vooght et al. 2018). Another strategy uses transgenic approaches to make Anopheles mosquitoes refractory to Plasmodium infection (Ito et al. 2002; Moreira et al. 2004; Meredith et al. 2011; Isaacs et al. 2012; Smith et al. 2013; Sumitani et al. 2013; McArthur et al. 2014; Gantz et al. 2015). For population replacement approaches, the plan is to use gene drives to spread and establish such a transgenic refractory system in a wild population. Synthetic gene drives, based on naturally occurring selfish genes (the so-called homing endonuclease genes (HEG)), were first proposed in 2003 (Burt 2003). HEGs can be reprogrammed to target other than their natural target sites in the genome, e.g. in the human malaria vector An. gambiae (Windbichler et al. 2011). However, reprogramming HEGs is a challenging process, and limits their application in developing new gene drives (Champer et al. 2016).

The discovery of CRISPR/Cas not only provided a universal genome editing tool but also opened the door for the fast development of gene-drive systems. Due to the easy reprogramming of the CRISPR/Cas target site via the sgRNA sequence, the CRISPR-derived gene drives could theoretically be used to engineer almost any trait. In fact, a CRISPR/Cas gene drive with dual anti-Plasmodium effector genes has already been created for the malaria mosquito An. stephensi (Gantz et al. 2015). In case population replacement is not the desired strategy, gene drives could also be used to temporarily or locally suppress a population, e.g. by targeting female reproduction as shown for An. gambiae (Hammond et al. 2016) or by female to male conversion as modelled for C. capitata (KaramiNejadRanjbar et al. 2018). The difference to other population suppression strategies, such as the SIT or the RIDL system, is that theoretically the gene drive requires only a single seed release of a small number of individuals spreading into the population in contrast to the necessity of repeated mass-releases of sterile or transgenic males.

\subsection{Mutations and Resistance Development to Genetic Modifications}

All genetically-based technologies have the risk that mutations and recombinations could alter or inactivate the transgene function. An important quality parameter for strains used in operational programmes is their genetic stability under large-scale rearing conditions. Mass-rearing facilities can produce hundreds or even thousands of millions of insects per week, which significantly increases the probability of the occurrence of these chance events (Dowell et al., this volume; Franz et al., this volume; Parker, Mamai et al., this volume;). Depending on where the mutations occur, they can have a variety of impacts on the transgene. Single-point mutations (which are the most likely mutations to occur) in the promoter or regulatory elements might not abolish the function of the element, but the accumulation of multiple mutations might do so. In contrast, a point mutation in a coding gene, leading to a missense or nonsense mutation, could cause a knockdown or knockout effect. The potential rate of a knockout mutation in a regulatory sequence can be estimated at the rate of $10^{-7}$ per bp or similar to a point-mutation reversion rate in a $\sim 1 \mathrm{~kb}$ coding region (Handler 2016), while a knockout mutation in a $1 \mathrm{~kb}$ coding region is estimated at a frequency of about $1-5 \times 10^{-6}$ (Tobari and Kojima 1972; Neel 1983; Woodruff et al. 1983). Therefore, under mass-rearing conditions (up to 
$2 \times 10^{9}$ insects per week), the occurrence of one or more revertant mutations can be expected. Upon release, these mutations might allow survival under restrictive conditions, and might even lead to population replacement or result in the breakdown of the mass-reared strain. Therefore, backup systems will be needed. Moreover, large-scale tests will be required to test for the actual occurrence of revertant mutations, and revertants to be analysed to identify the resistance mechanism. If a transgenic strain is mass-produced on a large scale, and taking into consideration natural mutation rates, a mutation inactivating the transgene resulting in genetic instability, and the breakdown of the transgenic strain, is possible (Handler 2016). This is a significant concern which has urged researchers in the field to consider strategies which can minimize or eliminate the likelihood of resistance development by incorporating, for example, a second selectable marker or effector gene (Eckermann et al. 2014; Handler 2016). However, such transgenic strains have not yet been developed and validated. Currently, filter rearing systems are used in mass-rearing to prevent spontaneous mutations from spreading in the mother colony and being released; each batch of insects is started from a checked population and then mass-reared for the release population (Parker, Mamai et al., this volume).

In the case of gene drives for population suppression or population replacement, it is expected that drive-resistant alleles will emerge for virtually any gene drive described so far (Champer et al. 2017; Noble et al. 2017; Unckless et al. 2017; KaramiNejadRanjbar et al. 2018). Resistance can arise from a variety of mechanisms. The most frequent reason will probably be by the action of the drive itself, when the Cas-induced double-strand break is not repaired by homologydirected repair as intended, but instead by the cell's non-homologous end-joining (NHEJ) repair mechanism, that often introduces small insertions or deletions at the repair site. These base changes will then not be recognized by the drive anymore, thus preventing the construct from spreading to the entire population. This is more likely to happen if the drive cuts only at one site. Other mechanisms of resistance are possible as well but will likely be a less frequent cause, such as standing genetic variation between populations as found for An. gambiae mosquitoes in Africa (Miles et al. 2016) or de novo mutations in the targeted gene(s), particularly if the target locus is not highly conserved.

Several solutions have been suggested to prevent resistance development against gene drives, e.g. targeting multiple sequences within a gene or targeting different genes at once, so that resistance would have to evolve independently for each site (Esvelt et al. 2014; Champer et al. 2016; Noble et al. 2017; KaramiNejadRanjbar et al. 2018). Moreover, it should be possible to prevent, or at least delay, the emergence of resistance by targeting highly conserved genes for which resistance due to mutation is expected to have a severe fitness cost, or to target a haplolethal gene whose function is only preserved under successful conversion (Esvelt et al. 2014; Champer et al. 2016; Noble et al. 2017). The concomitant expression of a short-hairpin RNA (shRNA) gene to suppress NHEJ repair could also help to prevent resistance development (Chu et al. 2015). 


\subsection{Regulatory Considerations for the Application of Transgenic Insects}

Most modern molecular techniques applied to insect genomes create transgenic insects. The use of transgenic strains in open-field conditions, and potentially in operational programmes, requires them to be of adequate biological quality, but they also need to have all of the necessary regulatory approvals (Reeves et al. 2012). It is worth noting that the regulatory framework for transgenic insects, developed by the various technologies, is not harmonized within and between different countries and continents, and this is not expected to change in the near future. In addition, scientists and managers planning to use transgenic insects in the field must, in advance, address questions and concerns raised by the general public and stakeholders. In that respect, a decision-making process based on scientific evidence, evaluations, risk assessment studies, and large-scale experiments (in the laboratory, but also in contained and open-field release trials) would be necessary (FAO/IPPC 2004; FAO/IAEA 2006). Thanks to the novel technologies, and because of the extremely different control scenarios unfolding, a clear case-by-case evaluation is needed. A generalized treatment of the different systems and/or insect strains should be avoided. Several efforts have been initiated to include transgenic insects in regulatory frameworks, e.g. by the North American Plant Protection Organization (NAPPO 2007), the European Food Safety Authority (EFSA 2013), and recently by the National Academy of Sciences, Engineering, and Medicine (NASEM 2016). These documents inform in a detailed and scientific way, and hopefully will foster a balanced discussion to improve the decision-making in this exciting but complicated modern biotechnological field.

As an alternative, non-transgenic approaches could be pursued. In addition to classical genetic approaches, CRISPR/Cas and RNAi strategies offer different possibilities. Although organisms created by CRISPR/Cas genome editing will be treated under the GMO directive in the EU, based on a very recent decision of the European Court of Justice, CRISPR mutations that could also have been developed through traditional breeding methods, such as point mutations or small insertions or deletions, are classified as non-GM by the US Department of Agriculture and the Government of Japan (Nature 2018). As the evaluation of the CRISPR technology by governments and authorities is just starting, in future CRISPR mutations have the potential to be classified as non-GM in more countries. Thus, in these countries, strains created by CRISPR mutagenesis would not be affected by the regulations for transgenic organisms. Such mutations could be used, for example, to knock out genes required for sex determination to create non-transgenic sexing strains. RNAi methods also offer a way to work without transgenic organisms, i.e. if the dsRNA is not conditionally or constitutively expressed in the insect, and if it does not persist in the environment. 


\subsection{Novel Developments in Insect Pest Control: Impaired Male Fertility}

Very recently, a completely new path to population suppression via impaired male fertility has been studied; the idea is based on the fact that mitochondria are inherited maternally. Therefore, mutations on the mitochondrial DNA (mtDNA) that are deleterious to males escape evolutionary selection to a high degree; the mutations don't have negative effects on the females which pass them on across generations (Frank and Hurst 1996; Gemmell et al. 2004; Beekman et al. 2014). By releasing females carrying such mutations into wild populations, these females and their female descendants would continuously produce males with impaired fertility, which should cause perpetual suppression of the target population. This idea was termed the Trojan Female Technique (TFT) (Gemmell et al. 2013). The initial data from different organisms, including Drosophila (Yee et al. 2013; Dowling et al. 2015; Patel et al. 2016; Wolff et al. 2016) and the cowpea weevil Callosobruchus maculatus (F.) (Dowling et al. 2007), show that such mtDNA mutations indeed can affect male fertility without affecting female fertility, supporting the possibility of using TFT for population suppression. However, there are many factors that could influence the effectiveness of the TFT (Wolff et al. 2016); for example, females could still get enough viable sperm to fertilize the eggs if males are not completely infertile or by remating with another partially fertile male. Moreover, the selective pressure on the males could lead to compensatory mutations, and genetic drift and population dynamics among other factors could lead to the disappearance of male fertility-impairing mutations even if they are not selected against (Rand et al. 2001; White et al. 2008).

An initial study with Drosophila melanogaster showed that a male-harming mitochondrial haplotype can indeed decrease the population size under densitycontrolled conditions over 10 generations by an average of $8 \%$ at a $75 \%$ haplotype frequency at the start of the treatment (Wolff et al. 2016). However, when the population size fluctuated, no effect was observed, although the TFT haplotype was stably persistent in most cases. Many more studies with different candidate mutations will be necessary to test if such Trojan females can indeed decrease the population size to meaningful levels under natural population conditions and across generations. If a naturally occurring mtDNA mutation was used, TFT would also be considered a non-transgenic approach.

Nevertheless, independent of the technology and strategy used, each product resulting from the application of modern biotechnology should be thoroughly tested and evaluated for functionality, efficacy, safety, and stability of the applied system. The ultimate goal is to select a safe, economical, and ethical strategy to perform environment-friendly and thus sustainable pest control for most of the important insect pest and vector species. 


\section{REFERENCES}

Andreasen, M. H., and C. F. Curtis. 2005. Optimal life stage for radiation sterilization of Anopheles males and their fitness for release. Medical and Veterinary Entomology 19: 238-244. http://onlinelibrary.wiley.com/doi/10.1111/j.1365-2915.2005.00565.x/full

Andrews, B. J., L. G. Beatty, and P. D. Sadowski. 1986. Site-specific recombination of the yeast plasmid two-micron circle: intermediates in the binding process. Basic Life Sciences 40: 407-424. https://link.springer.com/chapter/10.1007\%2F978-1-4684-5251-8_31

Ant, T., M. Koukidou, P. Rempoulakis, H. F. Gong, A. Economopoulos, J. Vontas, and L. Alphey. 2012. Control of the olive fruit fly using genetics-enhanced sterile insect technique. BMC Biology 10: 51. https://doi.org/10.1186/1741-7007-10-51

Araujo, R.N., A. Santos, F. S. Pinto, N. F. Gontijo, M. J. Lehane, and M. H. Pereira. 2006. RNA interference of the salivary gland nitrophorin 2 in the triatomine bug Rhodnius prolixus (Hemiptera: Reduviidae) by dsRNA ingestion or injection. Insect Biochemistry and Molecular Biology 36: 683693. https://doi.org/10.1016/j.ibmb.2006.05.012

Arnould, S., P. Chames, C. Perez, E. Lacroix, A. Duclert, J. C. Epinat, F. Stricher, A. S. Petit, A. Patin, S. Guillier, S. Rolland, J. Prieto, F. J. Blanco, J. Bravo, G. Montoya, L. Serrano, P. Duchateau, and F. Pâques. 2006. Engineering of large numbers of highly specific homing endonucleases that induce recombination on novel DNA targets. Journal of Molecular Biology 355: 443-458. DOI: 10.1016/j.jmb.2005.10.065 https://doi.org/10.1016/j.jmb.2005.10.065

Aryan, A., M. A. E. Anderson, K. M. Myles, and Z. N. Adelman. 2013. TALEN-based gene disruption in the dengue vector Aedes aegypti. PLOS ONE 8(3): e60082. https://doi.org/10.1371/journal.pone.0060082

Aryan, A., M. Anderson, J. K. Biedler, Y. Qi, J. M. Overcash, A. N. Naumenko, M. V. Sharakhova, C. Mao, Z. N. Adelman, Z. Tu. 2019. Nix confers heritable sex-conversion in Aedes aegypti and myo-sex is needed for male flight. bioRxiv. https://www.biorxiv.org/content/10.1101/595371v1

Augustinos, A. A., A. Targovska, E. Cancio-Martinez, E. Schom, G. Franz, C. Cáceres, A. Zacharopoulou, and K. Bourtzis. 2017. Ceratitis capitata genetic sexing strains: laboratory evaluation of strains from mass-rearing facilities worldwide. Entomologia Experimentalis et Applicata 164: 305-317. http://onlinelibrary.wiley.com/doi/10.1111/eea.12612/abstract

Aumann, R. A., M. F. Schetelig, and I. Häcker. 2018. Highly efficient genome editing by homologydirected repair using Cas9 protein in Ceratitis capitata. Insect Biochemistry and Molecular Biology 101: 85-93. https://doi.org/10.1016/j.ibmb.2018.08.004

Awata, H., T. Watanabe, Y. Hamanaka, T. Mito, S. Noji, and M. Mizunami. 2015. Knockout crickets for the study of learning and memory: dopamine receptor Dop1 mediates aversive but not appetitive reinforcement in crickets. Scientific Reports 5: 15885. https://www.nature.com/articles/srep15885

Baer, A., and J. Bode. 2001. Coping with kinetic and thermodynamic barriers: RMCE, an efficient strategy for the targeted integration of transgenes. Current Opinion in Biotechnology 12: 473-480. DOI: S0958-1669(00)00248-2 https://doi.org/10.1016/S0958-1669(00)00248-2

Bassett, A. R., C. Tibbit, C. P. Ponting, and J. L. Liu. 2014. Mutagenesis and homologous recombination in Drosophila cell lines using CRISPR/Cas9. Biology Open. 3: 42-49. http://bio.biologists.org/content/3/1/42

Bateman, J. R., A. M. Lee, and C. T. Wu. 2006. Site-specific transformation of Drosophila via phiC31 integrase-mediated cassette exchange. Genetics 173: 769-777. http://www.genetics.org/content/173/2/769

Baum, J. A., and J. K. Roberts. 2014. Progress towards RNAi-mediated insect pest management, chapter 5, pp. 249-295. In T. S. Dhadialla and S. S. Gill (eds.), Insect midgut and insecticidal proteins. Adv. in Insect Physiology (vol. 47). https://doi.org/10.1016/B978-0-12-800197-4.00005-1

Baum, J. A., T. Bogaert, W. Clinton, G. R. Heck, P. Feldmann, O. Ilagan, S. Johnson, G. Plaetinck, T. Munyikwa, M. Pleau, T. Vaughn, and J. Roberts. 2007. Control of coleopteran insect pests through RNA interference. Nature Biotechnology 25: 1322-1326. http://www.nature.com/nbt/journal/v25/n11/full/nbt1359.html

Beekman, M., D. K. Dowling, and D. K. Aanen. 2014. The costs of being male: are there sex-specific effects of uniparental mitochondrial inheritance? Philos. Trans. Royal Soc. B Biol. Sci. 369(1646): 20130440. http://rstb.royalsocietypublishing.org/content/369/1646/20130440 
Bellini, R., A. Puggioli, F. Balestrino, M. Carrieri, and S. Urbanelli. 2018. Exploring protandry and pupal size selection for Aedes albopictus sex separation. Parasites and Vectors 11(Suppl. 2): 650. https://doi.org/10.1186/s13071-018-3213-x

Bello, B., D. Resendez-Perez, and W. J. Gehring. 1998. Spatial and temporal targeting of gene expression in Drosophila by means of a tetracycline-dependent transactivator system. Development 125: 2193-2202. https://www.ncbi.nlm.nih.gov/pubmed/9584119

Ben Ami, E., B. Yuval, and E. Jurkevitch. 2010. Manipulation of the microbiota of mass-reared Mediterranean fruit flies Ceratitis capitata (Diptera: Tephritidae) improves sterile male sexual performance. The ISME Journal 4: 28-37. http://www.nature.com/articles/ismej200982

Bernardini, F., R. Galizi, M. Menichelli, P. A. Papathanos, V. Dritsou, E. Marois, A. Crisanti, and N. Windbichler. 2014. Site-specific genetic engineering of the Anopheles gambiae Y chromosome. Proc. Nat. Acad. Sci. USA 111(21): 7600-7605. http://www.pnas.org/content/111/21/7600.abstract

Bhaya, D., M. Davison, and R. Barrangou. 2011. CRISPR-Cas systems in bacteria and archaea: versatile small RNAs for adaptive defense and regulation. Annual Review of Genetics 45: 273-297. https://doi.org/10.1146/annurev-genet-110410-132430

Bi, H. L., J. Xu, A. J. Tan, and Y. P. Huang. 2016. CRISPR/Cas9-mediated targeted gene mutagenesis in Spodoptera litura. Insect Science 23: 469-477. http://onlinelibrary.wiley.com/doi/10.1111/1744-7917.12341/abstract

Bloem, S., J. E. Carpenter, K. A. Bloem, L. Tomlin, and S. Taggart. 2004. Effect of rearing strategy and gamma radiation on field competitiveness of mass-reared codling moths (Lepidoptera: Tortricidae). Journal of Economic Entomology 97: 1891-1898. https://doi.org/10.1093/jee/97.6.1891

Bode, J., T. Schlake, M. Iber, D. Schübeler, J. Seibler, E. Snezhkov, and L. Nikolaev. 2000. The transgeneticist's toolbox: novel methods for the targeted modification of eukaryotic genomes. Biological Chemistry 381: 801-813. https://www.degruyter.com/view/j/bchm.2000.381.issue-9-10/bc.2000.103/bc.2000.103.xml

Bond, J. G., A. Ramírez-Osorio, C. F. Marina, I. Fernández-Salas, P. Liedo, A. Dor, and T. Williams. 2017. Efficiency of two larval diets for mass-rearing of the mosquito Aedes aegypti. PLOS ONE 12(11): e0187420. https://doi.org/10.1371/journal.pone.0187420

Bourtzis, K., H. R. Braig, and T. L. Karr. 2003. Cytoplasmic incompatibility, pp. 217-246. In K. Bourtzis and T. A. Miller (eds.), Insect symbiosis. CRC Press, Boca Raton, FL, USA. https://www.crcpress.com/Insect-Symbiosis/Bourtzis-Miller/p/book/9780849312861

Bourtzis, K., S. L. Dobson, Z. Xi, J. L. Rasgon, M. Calvitti, L. A. Moreira, H. C. Bossin, R. Moretti, L. A. Baton, G. L. Hughes, P. Mavingui, and J. R. L. Gilles. 2014. Harnessing mosquitoWolbachia symbiosis for vector and disease control. Acta Tropica 132 Suppl.: S150-S163. https://doi.org/10.1016/j.actatropica.2013.11.004

Bourtzis, K., R. S. Lees, J. Hendrichs, and M. J. B. Vreysen. 2016. More than one rabbit out of the hat: radiation, transgenic and symbiont-based approaches for sustainable management of mosquito and tsetse fly populations. Acta Tropica $157: 115-130$.

https://doi.org/10.1016/j.actatropica.2016.01.009

Bozas, A., K. J. Beumer, J. K. Trautman, and D. Carroll. 2009. Genetic analysis of zinc-finger nuclease-induced gene targeting in Drosophila. Genetics 182: 641-651. http://www.genetics.org/content/182/3/641

Brand, A. H., and N. Perrimon. 1993. Targeted gene expression as a means of altering cell fates and generating dominant phenotypes. Development 118: 401-415. https://www.ncbi.nlm.nih.gov/pubmed/8223268

Bucher, G., J. Scholten, and M. Klingler. 2002. Parental RNAi in Tribolium (Coleoptera). Current Biology 12(3): R85-R86. https://doi.org/10.1016/S0960-9822(02)00666-8

Burt, A. 2003. Site-specific selfish genes as tools for the control and genetic engineering of natural populations. Proceedings of the Royal Society B: Biological Sciences 270(1518): 921-928. http://rspb.royalsocietypublishing.org/content/270/1518/921

Cáceres, C., J. P. Cayol, W. Enkerlin, G. Franz, J. Hendrichs, and A. S. Robinson. 2004. Comparison of Mediterranean fruit fly (Ceratitis capitata) (Tephritidae) bisexual and genetic sexing strains: development, evaluation and economics, pp. 367-381. In B. N. Barnes (ed.), Proceedings of the 6th International Symposium on Fruit Flies of Economic Importance, 6-10 May 2002, Stellenbosch, South Africa. Isteg Scientific Publications, Irene, South Africa. https://nucleus.iaea.org/sites/naipc/twd/Documents/6thISFFEI_Proceedings/6th-ISFFEI-Proceedings2004-Stellenbosch-South-Africa.pdf 
Callaway, E. 2016. Infected mosquitoes fight Zika. South America hosts largest trials yet of Wolbachiainfected insects to combat viruses. Nature 539(7627): 17-18. DOI: 10.1038/nature.2016.20878 https://www.nature.com/polopoly_fs/1.20878!/menu/main/topColumns/topLeftColumn/pdf/nature.20 16.20878.pdf

Carvalho, D. O., D. Nimmo, N. Naish, A. R. McKemey, P. Gray, A. B. B. Wilke, M. T. Marrelli, J. F. Virginio, L. Alphey, and M. L. Capurro. 2014. Mass production of genetically modified Aedes aegypti for field releases in Brazil. Journal of Visualized Experiments 83: e3579.

https://www.jove.com/video/3579/mass-production-genetically-modified-aedes-aegypti-for-fieldreleases

Carvalho, D. O., A. R. McKemey, L. Garziera, R. Lacroix, C. A. Donnelly, L. Alphey, A. Malavasi, and M. L. Capurro. 2015. Suppression of a field population of Aedes aegypti in Brazil by sustained release of transgenic male mosquitoes. PLOS Neglected Tropical Diseases 9(7): e0003864. https://doi.org/10.1371/journal.pntd.0003864

Cary, L. C., M. Goebel, B. G. Corsaro, H. G. Wang, E. Rosen, and M. J. Fraser. 1989. Transposon mutagenesis of baculoviruses: analysis of Trichoplusia ni transposon IFP2 insertions within the FPlocus of nuclear polyhedrosis viruses. Virology 172: 156-169. https://www.ncbi.nlm.nih.gov/pubmed/2549707

Catteruccia, F., H. C. Godfray, and A. Crisanti. 2003. Impact of genetic manipulation on the fitness of Anopheles stephensi mosquitoes. Science 299(5610): 1225-1227. https://doi.org/10.1126/science. 1081453

Catteruccia, F., J. P. Benton, and A. Crisanti. 2005. An Anopheles transgenic sexing strain for vector control. Nature Biotechnology 23: 1414-1417. http://www.nature.com/nbt/journal/v23/n11/full/nbt1152.html?foxtrotcallback=true

(CBD) Convention on Biological Diversity. 2000. Cartegena Protocol on Biosafety to the Convention on Biological Diversity. https://www.cbd.int/doc/legal/cartagena-protocol-en.pdf

(CBD) Convention on Biological Diversity. 2007. Glossary of terms for negotiators of multilateral environmental agreements. https://www.cbd.int/doc/guidelines/MEAs-negotiator-glossary-terms-en.pdf

Champer, J., A. Buchman, and O. S. Akbari. 2016. Cheating evolution: engineering gene drives to manipulate the fate of wild populations. Nature Reviews Genetics 17: 146-159. http://www.nature.com/nrg/journal/v17/n3/abs/nrg.2015.34.html

Champer, J., R. Reeves, S. Y. Oh, C. Liu, J. Liu, A. G. Clark, and P. W. Messer. 2017. Novel CRISPR/Cas9 gene drive constructs reveal insights into mechanisms of resistance allele formation and drive efficiency in genetically diverse populations. PLOS Genetics 13(7):e1006796. https://doi.org/10.1371/journal.pgen.1006796

Chu, V. T., T. Weber, B. Wefers, W. Wurst, S. Sander, K. Rajewsky, and R. Kühn. 2015. Increasing the efficiency of homology-directed repair for CRISPR-Cas9-induced precise gene editing in mammalian cells. Nature Biotechnology 33: 543-548.

http://www.nature.com/nbt/journal/v33/n5/full/nbt.3198.html

Coates, C. J., J. M. Kaminski, J. B. Summers, D. J. Segal, A. D. Miller, and A. F. Kolb. 2005. Sitedirected genome modification: derivatives of DNA-modifying enzymes as targeting tools. Trends in Biotech. 23: 407-419. http://www.cell.com/trends/biotechnology/abstract/S0167-7799(05)00170-8

Concha, C., and M. J. Scott. 2009. Sexual development in Lucilia cuprina (Diptera, Calliphoridae) is controlled by the transformer gene. Genetics 182: 785-798. https://doi.org/10.1534/genetics.109.100982

Concha, C., A. Palavesam, F. D. Guerrero, A. Sagel, F. Li, J. A. Osborne, Y. Hernandez, T. Pardo, G. Quintero, M. Vasquez, G. P. Keller, P. L. Phillips, J. B. Welch, W. O. McMillan, S. R. Skoda, and M. J. Scott. 2016. A transgenic male-only strain of the New World screwworm for an improved control program using the sterile insect technique. BMC Biology 14: 72. https://bmcbiol.biomedcentral.com/articles/10.1186/s12915-016-0296-8

Cooper, A. M. W., K. Silver, J. Zhang, Y. Park, and K. Y. Zhu. 2019. Molecular mechanisms influencing efficiency of RNA interference in insects. Pest Management Science 75: 18-28. https://doi.org/10.1002/ps.5126

Criscione, F., Y. Qi, R. Saunders, B. Hall, and Z. Tu. 2013. A unique Y gene in the Asian malaria mosquito Anopheles stephensi encodes a small lysine-rich protein and is transcribed at the onset of embryonic development. Insect Molecular Biology 22: 433-441. https://doi.org/10.1111/imb.12034

Criscione, F., Y. Qi, and Z. Tu. 2016. GUY1 confers complete female lethality and is a strong candidate for a male-determining factor in Anopheles stephensi. eLife 5: e19281. 
https://dx.doi.org/10.7554/eLife.19281.001

Dafa'alla, T. H., G. C. Condon, K. C. Condon, C. E. Phillips, N. I. Morrison, L. Jin, M. J. Epton, G. Fu, and L. Alphey. 2006. Transposon-free insertions for insect genetic engineering. Nature Biotechnology 24: 820-821. http://www.nature.com/nbt/journal/v24/n7/full/nbt1221.html

Darrington, M., T. Dalmay, N. I. Morrison, and T. Chapman. 2017. Implementing the sterile insect technique with RNA interference - a review. Entomologia Experimentalis et Applicata 164: 155-175. http://onlinelibrary.wiley.com/doi/10.1111/eea.12575/full

De Vooght, L., S. Van Keer, and J. Van Den Abbeele. 2018. Towards improving tsetse fly paratransgenesis: stable colonization of Glossina morsitans morsitans with genetically modified Sodalis. BMC Microbiology 18(Suppl. 1):165. https://doi.org/10.1186/s12866-018-1282-9

Dow, J. A. 1992. pH gradients in lepidopteran midgut. Journal of Experimental Biology 172: 355-375. http://jeb.biologists.org/content/172/1/355

Dowling, D. K., A. Larkeson Nowostawski, and G. Arnqvist. 2007. Effects of cytoplasmic genes on sperm viability and sperm morphology in a seed beetle: implications for sperm competition theory? J. Evol. Biol. 20: 358-368. http://onlinelibrary.wiley.com/doi/10.1111/j.1420-9101.2006.01189.x/full

Dowling, D. K., D. M. Tompkins, and N. J. Gemmell. 2015. The Trojan Female Technique for pest control: a candidate mitochondrial mutation confers low male fertility across diverse nuclear backgrounds in Drosophila melanogaster. Evolutionary Applications 8: 871-880. http://onlinelibrary.wiley.com/doi/10.1111/eva.12297/full

Dutra, H. L., M. N. Rocha, F. B. Dias, S. B. Mansur, E. P. Caragata, and L. A. Moreira. 2016. Wolbachia blocks currently circulating Zika virus isolates in Brazilian Aedes aegypti mosquitoes. Cell Host Microbe 19: 771-774. https://www.ncbi.nlm.nih.gov/pubmed/27156023

Eckermann, K. N., S. Dippel, M. KaramiNejadRanjbar, H. M. Ahmed, I. M. Curril, and E. A. Wimmer. 2014. Perspective on the combined use of an independent transgenic sexing and a multifactorial reproductive sterility system to avoid resistance development against transgenic sterile insect technique approaches. BMC Genetics 15(Suppl. 2): S17. https://www.ncbi.nlm.nih.gov/pubmed/25471733

Eckermann, K. N., H. M. M. Ahmed, M. KaramiNejadRanjbar, S. Dippel, C. E. Ogaugwu, P. Kitzmann, M. D. Isah, and E. A. Wimmer. 2018. Hyperactive piggyBac transposase improves transformation efficiency in diverse insect species. Insect Biochemistry and Molecular Biology 98: 16-24. https://doi.org/10.1016/j.ibmb.2018.04.001

(EFSA) European Food Safety Authority. 2013. Guidance on the environmental risk assessment of genetically modified animals. EFSA Panel on Genetically Modified Organisms (GMO). EFSA Journal 11(5): 3200. https://efsa.onlinelibrary.wiley.com/doi/epdf/10.2903/j.efsa.2013.3200

Elick, T. A., C. A. Bauser, N. M. Principe, and M. J. Fraser, Jr. 1996. PCR analysis of insertion site specificity, transcription, and structural uniformity of the Lepidopteran transposable element IFP2 in the TN-368 cell genome. Genetica 97(2): 127-139. https://www.ncbi.nlm.nih.gov/pubmed/8901134

Esvelt, K. M., A. L. Smidler, F. Catteruccia, and G. M. Church. 2014. Concerning RNA-guided gene drives for the alteration of wild populations. eLife 3. pii: e03401. DOI: 10.7554/eLife.03401. https://www.ncbi.nlm.nih.gov/pubmed/25035423

Evans, B. R., P. Kotsakiozi, A. L. Costa-da-Silva, R. S. Ioshino, L. Garziera, M. C. Pedrosa, A. Malavasi, J. F. Virginio, M. L. Capurro, and J. R. Powell. 2019. Transgenic Aedes aegypti mosquitoes transfer genes into a natural population. Scientific Reports (Nature Research) 9: 13047. https://doi.org/10.1038/s41598-019-49660-6

Facchinelli, L., L.Valerio, J. M. Ramsey, F. Gould, R. K. Walsh, G. Bond, M. A. Robert, A. L. Lloyd, A. A. James, L. Alphey, and T. W. Scott. 2013. Field cage studies and progressive evaluation of genetically-engineered mosquitoes. PLOS Neglected Tropical Diseases 7(1):e2001. http://journals.plos.org/plosntds/article?id=10.1371/journal.pntd.0002001

(FAO/IAEA) Food and Agriculture Organization of the United Nations/International Atomic Energy Agency. 2006. Status and risk assessment of the use of transgenic arthropods in plant protection. Proceedings of a Technical Meeting Held in Rome, 8-12 May 2002. IAEA-TECDOC1483. FAO/IAEA, Vienna, Austria. 155 pp. https://www.iaea.org/publications/7204/status-and-riskassessment-of-the-use-of-transgenic-arthropods-in-plant-protection

(FAO/IAEA/USDA) Food and Agriculture Organization of the United Nations/International Atomic Energy Agency/United States Department of Agriculture. 2019. Product quality control for sterile mass-reared and released tephritid fruit flies. Version 7.0. IAEA, Vienna, Austria. 148 pp. https://www.iaea.org/sites/default/files/qcv7.pdf 
(FAO/IPPC) Food and Agriculture Organization of the United Nations/International Plant Protection Convention. 2004. Pest risk analysis for quarantine pests including analysis of environmental risks and living modified organisms. International Standard for Phytosanitary Measures (ISPM) No. 11. http://www.furs.si/law/FAO/ZVR/ENG/sprISPM_11_2004.pdf

(FAO/IPPC) Food and Agriculture Organization of the United Nations/International Plant Protection Convention. 2017. Glossary of phytosanitary terms, ISPM 5. https://www.ippc.int/en/publications/glossary-phytosanitary-terms/

Frank, S. A., and L. D. Hurst. 1996. Mitochondria and male disease. Nature 383(6597): 224. DOI: $10.1038 / 383224 \mathrm{a} 0 \mathrm{http}: / /$ www.nature.com/articles/383224a0

Franz, A. W., N. Jasinskiene, I. Sanchez-Vargas, A. T. Isaacs, M. R. Smith, C. C. Khoo, M. S. Heersink, A. A. James, and K. E. Olson. 2011. Comparison of transgene expression in Aedes aegypti generated by mariner Mos1 transposition and PhiC31 site-directed recombination. Insect Molecular Biology 20: 587-598. https://www.ncbi.nlm.nih.gov/pubmed/21699593

Franz, G., and D. O. McInnis. 1995. A promising new twist-a genetic sexing strain based on a temperature sensitive lethal (tsl) mutation, pp. 187-196. In The Mediterranean fruit fly in California: defining critical research. Riverside: University of California, USA.

Franz, G., and C. Savakis. 1991. Minos, a new transposable element from Drosophila hydei, is a member of the Tc1-like family of transposons. Nucleic Acids Research 19(23): 6646. https://www.ncbi.nlm.nih.gov/pmc/articles/PMC329244/?page=1

Fu, G., K. C. Condon, M. J. Epton, P. Gong, L. Jin, G. C. Condon, N. I. Morrison, T. H. Dafa'alla, and L. Alphey. 2007. Female-specific insect lethality engineered using alternative splicing. Nature Biotechnology 25: 353-357.

http://www.nature.com/nbt/journal/v25/n3/full/nbt1283.html?foxtrotcallback=true

Fu, Y., J. D. Sander, D. Reyon, V. M. Cascio, and J. K. Joung. 2014. Improving CRISPR-Cas nuclease specificity using truncated guide RNAs. Nature Biotechnology 32: 279-284. http://www.nature.com/nbt/journal/v32/n3/full/nbt.2808.html

Funaguma, S., M. G. Suzuki, T. Tamura, and T. Shimada. 2005. The $B m d s x$ transgene including trimmed introns is sex-specifically spliced in tissues of the silkworm, Bombyx mori. Journal of Insect Science 5: 17. https://doi.org/10.1093/jis/5.1.17

Galizi, R., L. A. Doyle, M. Menichelli, F. Bernardini, A. Deredec, A. Burt, B. L. Stoddard, N. Windbichler, and A. Crisanti. 2014. A synthetic sex ratio distortion system for the control of the human malaria mosquito. Nature Comm. 5: 3977. https://www.nature.com/articles/ncomms4977

Galizi, R., A. Hammond, K. Kyrou, C. Taxiarchi, F. Bernardini, S. M. O'Loughlin, P. A. Papathanos, T. Nolan, N. Windbichler, and A. Crisanti. 2016. A CRISPR-Cas9 sex-ratio distortion system for genetic control. Scientific Reports 6: 31139. DOI: 10.1038/srep31139 https:/www.ncbi.nlm.nih.gov/pmc/articles/PMC4971495/

Gantz, V. M., N. Jasinskiene, O. Tatarenkova, A. Fazekas, V. M. Macias, E. Bier, and A. A. James. 2015. Highly efficient Cas9-mediated gene drive for population modification of the malaria vector mosquito Anopheles stephensi. Proceedings of the National Academy of Sciences USA 112(49):E6736-43. http://www.pnas.org/content/112/49/E6736.abstract

Garbutt, J. S., X. Bellés, E. H. Richards, and S. E. Reynolds. 2013. Persistence of double-stranded RNA in insect hemolymph as a potential determiner of RNA interference success: evidence from Manduca sexta and Blattella germanica. Journal of Insect Physiology 59(2): 171-178. https://www.ncbi.nlm.nih.gov/pubmed/22664137

Garziera, L., M. C. Pedrosa, F. A. de Souza, M. Gómez, M. B. Moreira, J. F. Virginio, M. L. Capurro, and D. O. Carvalho. 2017. Effect of interruption of over-flooding releases of transgenic mosquitoes over wild population of Aedes aegypti: two case studies in Brazil. Entomologia Experimentalis et Applicata 164: 327-339. http://onlinelibrary.wiley.com/doi/10.1111/eea.12618/full

Gemmell, N. J., V. J. Metcalf, and F. W. Allendorf. 2004. Mother's curse: the effect of mtDNA on individual fitness and population viability. Trends in Ecology and Evolution 19: 238-244. https://doi.org/10.1016/j.tree.2004.02.002

Gemmell, N. J., A. Jalilzadeh, R. K. Didham, T. Soboleva, and D. M. Tompkins. 2013. The Trojan female technique: a novel, effective and humane approach for pest population control. Proceedings of the Royal Society B 280(1773): 20132549. http://dx.doi.org/10.1098/rspb.2013.2549

Geuverink, E., and L. W. Beukeboom. 2014. Phylogenetic distribution and evolutionary dynamics of the sex determination genes doublesex and transformer in insects. Sexual Development 8(1-3): 38 49. DOI: 10.1159/000357056. https://www.karger.com/Article/FullText/357056 
Gilles, J. R. L., M. F. Schetelig, F. Scolari, F. Marec, M. L. Capurro, G. Franz, and K. Bourtzis. 2014. Towards mosquito sterile insect technique programmes: exploring genetic, molecular, mechanical and behavioural methods of sex separation in mosquitoes. Acta Tropica 132 Suppl. S178-S187. https://doi.org/10.1016/j.actatropica.2013.08.015

Gong, P., M. J. Epton, G. Fu, S. Scaife, A. Hiscox, K. C. Condon, G. C. Condon, N. I. Morrison, D. W. Kelly, T. Dafa'alla, P. G. Coleman, and L. Alphey. 2005. A dominant lethal genetic system for autocidal control of the Mediterranean fruitfly. Nature Biotechnology 23: 453-456. www.nature.com/articles/nbt1071

Gossen, M., and H. Bujard. 1992. Tight control of gene expression in mammalian cells by tetracyclineresponsive promoters. Proceedings of the National Academy of Sciences of the USA 89(12): 55475551. http://www.pnas.org/content/89/12/5547

Gossen, M., S. Freundlieb, G. Bender, G. Müller, W. Hillen, and H. Bujard. 1995. Transcriptional activation by tetracyclines in mammalian cells. Science 268(5218): 1766-1769. https://www.ncbi.nlm.nih.gov/pubmed/7792603

Guerfali, M. M., A. Parker, S. Fadhl, H. Hemdane, A. Raies, and C. Chevrier. 2011. Fitness and reproductive potential of irradiated mass-reared Mediterranean fruit fly males Ceratitis capitata (Diptera: Tephritidae): lowering radiation doses. Florida Entomologist 94: 1042-1050. https://doi.org/10.1653/024.094.0443

Häcker, I., and M. F. Schetelig. 2018. Molecular tools to create new strains for mosquito sexing and vector control. Parasites and Vectors 11(Suppl. 2): 645. https://doi.org/10.1186/s13071-018-3209-6

Häcker, I., R. A. Harrell II, G. Eichner, K. L. Pilitt, D. A. O'Brochta, A. M. Handler, and M. F. Schetelig. 2017. Cre/lox-recombinase-mediated cassette exchange for reversible site-specific genomic targeting of the disease vector, Aedes aegypti. Scientific Reports 7: 43883. https://doi.org/10.1038/srep43883

Haghighat-Khah, R. E., S. Scaife, S. Martins, O. St John, K. J. Matzen, N. Morrison, and L. Alphey. 2015. Site-specific cassette exchange systems in the Aedes aegypti mosquito and the Plutella xylostella moth. PLOS ONE 10(4): e0121097. http://journals.plos.org/plosone/article?id=10.1371/journal.pone.0121097

Hagler, J. R., and C. G. Jackson. 2001. Methods for marking insects: current techniques and future prospects. Annual Review of Entomology 46: 511-543. https://doi.org/10.1146/annurev.ento.46.1.511

Hall, A. B., V. A. Timoshevskiy, M. V. Sharakhova, X. Jiang, S. Basu, M. A. Anderson, W. Hu, I. V. Sharakhov, Z. N. Adelman, and Z. Tu. 2014. Insights into the preservation of the homomorphic sex-determining chromosome of Aedes aegypti from the discovery of a male-biased gene tightly linked to the M-locus. Genome Biology and Evolution 6: 179-191. https://www.ncbi.nlm.nih.gov/pubmed/24398378

Hall, A. B., S. Basu, X. Jiang, Y. Qi, V. A. Timoshevskiy, J. K. Biedler, M. V. Sharakhova, R. Elahi, M. A. E. Anderson, X. G. Chen, I. V. Sharakhov, Z. N. Adelman, and Z. Tu. 2015. A maledetermining factor in the mosquito Aedes aegypti. Science 348(6240): 1268-1270. http://science.sciencemag.org/content/348/6240/1268

Hamden, H., M. M. Guerfali, S. Fadhl, M. Saidi, and C. Chevrier. 2013. Fitness improvement of mass-reared sterile males of Ceratitis capitata (Vienna 8 strain) (Diptera: Tephritidae) after gut enrichment with probiotics. J. Economic Entomol. 106: 641-647. https://doi.org/10.1603/EC12362

Hammond, A., R. Galizi, K. Kyrou, A. Simoni, C. Siniscalchi, D. Katsanos, M. Gribble, D. Baker, E. Marois, S. Russell, A. Burt, N. Windbichler, A. Crisanti, and T. Nolan. 2016. A CRISPRCas 9 gene drive system targeting female reproduction in the malaria mosquito vector Anopheles gambiae. Nature Biotech. 34: 78-83. http://www.nature.com/nbt/journal/v34/n1/full/nbt.3439.html

Handler, A. M. 2002. Use of the piggyBac transposon for germ-line transformation of insects. Insect Biochemistry and Molecular Biology 32: 1211-1220. https://www.ars.usda.gov/arsuserfiles/2278/USE\%20OF\%20THE\%20PIGGYBAC\%202.pdf

Handler, A. M. 2004. Understanding and improving transgene stability and expression in insects for SIT and conditional lethal release programs. Insect Biochemistry and Molecular Biology 34: 121130. http://europepmc.org/abstract/med/14871608

Handler, A. M. 2016. Enhancing the stability and ecological safety of mass-reared transgenic strains for field release by redundant conditional lethality systems. Insect Science 23: 225-234. http://onlinelibrary.wiley.com/doi/10.1111/1744-7917.12245/abstract

Handler, A. M., and R. A. Harrell. 1999. Germline transformation of Drosophila melanogaster with the piggyBac transposon vector. Insect Molecular Biology 8: 449-457. 
http://onlinelibrary.wiley.com/doi/10.1046/j.1365-2583.1999.00139.x/abstract

Handler, A. M., and R. A. Harrell. 2001a. Transformation of the Caribbean fruit fly, Anastrepha suspensa, with a piggyBac vector marked with polyubiquitin-regulated GFP. Insect Biochemistry and Molecular Biology 31: 199-205. https://www.ncbi.nlm.nih.gov/pubmed/11164342

Handler, A. M., and R. A. Harrell. 2001b. Polyubiquitin-regulated DsRed marker for transgenic insects. Biotechniques 31: 820, 824-828. https://www.ncbi.nlm.nih.gov/pubmed/11680713

Handler, A. M., S. D. McCombs, M. J. Fraser, and S. H. Saul. 1998. The lepidopteran transposon vector, piggyBac, mediates germ-line transformation in the Mediterranean fruit fly. Proceedings of the National Academy of Sciences of the USA 95: 7520-7525. www.pnas.org/content/95/13/7520.f

Harris, A. F., D. Nimmo, A. R. McKemey, N. Kelly, S. Scaife, C. A. Donnelly, C. Beech, W. D. Petrie, and L. Alphey. 2011. Field performance of engineered male mosquitoes. Nature Biotechnology 29: 1034-1037. http://www.nature.com/nbt/journal/v29/n11/full/nbt.2019.html

Harris, A. F., A. R. McKemey, D. Nimmo, Z. Curtis, I. Black, S. A. Morgan, M. N. Oviedo, R. Lacroix, N. Naish, N. I. Morrison, A. Collado, J. Stevenson, S. Scaife, T. Dafa'alla, G. Fu, C. Phillips, A. Miles, N. Raduan, N. Kelly, C. Beech, C. A. Donnelly, W. D. Petrie, and L. Alphey. 2012. Successful suppression of a field mosquito population by sustained release of engineered male mosquitoes. Nature Biotechnology 30: 828-830.

http://www.nature.com/nbt/journal/v30/n9/abs/nbt.2350.html

Hayashi, S., K. Ito, Y. Sado, M. Taniguchi, A. Akimoto, H. Takeuchi, T. Aigaki, F. Matsuzaki, H. Nakagoshi, T. Tanimura, R. Ueda, T. Uemura, M. Yoshihara, and S. Goto. 2002. GETDB, a database compiling expression patterns and molecular locations of a collection of gal4 enhancer traps. Genesis (The Journal of Genetics and Development) 34: 58-61.

http://onlinelibrary.wiley.com/doi/10.1002/gene.10137/abstract

Heinrich, J. C., and M. J. Scott. 2000. A repressible female-specific lethal genetic system for making transgenic insect strains suitable for a sterile-release program. Proceedings of the National Academy of Sciences of the USA 97(15): 8229-8232. https://doi.org/10.1073/pnas.140142697

Hendrichs, J., G. Franz, and P. Rendon. 1995. Increased effectiveness and applicability of the sterile insect technique through male-only releases for control of Mediterranean fruit flies during fruiting seasons. Journal of Applied Entomology 119: 371-377. http://onlinelibrary.wiley.com/doi/10.1111/j.1439-0418.1995.tb01303.x/full

Hoess, R. H., A. Wierzbicki, and K. Abremski. 1986. The role of the loxP spacer region in P1 sitespecific recombination. Nucleic Acids Research 14(5): 2287-2300.

https:/www.ncbi.nlm.nih.gov/pmc/articles/PMC339658/

Hoffmann, A. A., B. L. Montgomery, J. Popovici, I. Iturbe-Ormaetxe, P. H. Johnson, F. Muzzi, M. Greenfield, M. Durkan, Y. S. Leong, Y. Dong, H. Cook, J. Axford, A. G. Callahan, N. Kenny, C. Omodei, E. A. McGraw, P. A. Ryan, S. A. Ritchie, M. Turelli, and S. L. O'Neill. 2011. Successful establishment of Wolbachia in Aedes populations to suppress dengue transmission. Nature 476: 454-457. http://www.nature.com/nature/journal/v476/n7361/full/nature10356.html

Hooper, G. H. S. 1972. Sterilization of the Mediterranean fruit fly with gamma radiation: effect on male competitiveness and change in fertility of females alternately mated with irradiated and untreated males. Journal of Economic Entomology 65: 1-6. https://doi.org/10.1093/jee/65.1.1

Horn, C., and A. M. Handler. 2005. Site-specific genomic targeting in Drosophila. Proc. National Academy of Sciences USA. 102(35): 12483-12488. http://www.pnas.org/content/102/35/12483

Horn, C., and E. A. Wimmer. 2003. A transgene-based, embryo-specific lethality system for insect pest management. Nature Biotechnology 21: 64-70. http://www.nature.com/nbt/journal/v21/n1/full/nbt769.html

Horvath, P., and R. Barrangou. 2010. CRISPR/Cas, the immune system of bacteria and archaea. Science 327(5962): 167-170. http://science.sciencemag.org/content/327/5962/167

Imamura, M., J. Nakai, S. Inoue, G. X. Quan, T. Kanda, and T. Tamura. 2003. Targeted gene expression using the GAL4/UAS system in the silkworm Bombyx mori. Genetics 165: 1329-1340. https://www.ncbi.nlm.nih.gov/pmc/articles/PMC1462818/

Irvin, N., M. S. Hoddle, D. A. O'Brochta, B. Carey, and P. W. Atkinson. 2004. Assessing fitness costs for transgenic Aedes aegypti expressing the GFP marker and transposase genes. Proc. Nat. Academy Sciences USA 101(3): 891-896. https://www.ncbi.nlm.nih.gov/pmc/articles/PMC321777

Isaacs, A. T., N. Jasinskiene, M. Tretiakov, I. Thiery, A. Zettor, C. Bourgouin, and A. A. James. 2012. Transgenic Anopheles stephensi coexpressing single-chain antibodies resist Plasmodium falciparum development. Proceedings of the National Academy of Sciences of the USA 109(28): E1922-E1930. http://www.pnas.org/content/109/28/E1922 
Isasawin, S., N. Aketarawong, and S. Thanaphum. 2012. Characterization and evaluation of microsatellite markers in a strain of the oriental fruit fly, Bactrocera dorsalis (Diptera: Tephritidae), with a genetic sexing character used in sterile insect population control. European Journal of Entomology 109: 331-338. https://www.eje.cz/artkey/eje-201203-0005.php

Isasawin, S., N. Aketarawong, S. Lertsiri, and S. Thanaphum. 2014. Development of a genetic sexing strain in Bactrocera carambolae (Diptera: Tephritidae) by introgression of sex sorting components from B. dorsalis, Salaya1 strain. BMC Genetics 15(Supp1 2): S2. https://doi.org/10.1186/1471-2156-15-S2-S2

Ito, J., A. Ghosh, L. A. Moreira, E. A. Wimmer, and M. Jacobs-Lorena. 2002. Transgenic anopheline mosquitoes impaired in transmission of a malaria parasite. Nature 417(6887): 452-455. https://doi.org/10.1038/417452a https://www.nature.com/articles/417452a

Ivashuta, S., Y. Zhang, B. E. Wiggins, P. Ramaseshadri, G. C. Segers, S. Johnson, S. E. Meyer, R. A. Kerstetter, B. C. McNulty, R. Bolognesi, and G. R. Heck. 2015. Environmental RNAi in herbivorous insects. RNA 21: 840-850. http://rnajournal.cshlp.org/content/21/5/840.full

Jacobson, J. W., M. M. Medhora, and D. L. Hartl. 1986. Molecular structure of a somatically unstable transposable element in Drosophila. Proceedings of National Academy of Sciences of the USA 83(22): 8684-8688. https://doi.org/10.1073/pnas.83.22.8684

Jin, L., A. S. Walker, G. Fu, T. Harvey-Samuel, T. Dafa'alla, A. Miles, T. Marubbi, D. Granville, N. Humphrey-Jones, S. O'Connell, N. I. Morrison, and L. Alphey. 2013. Engineered femalespecific lethality for control of pest Lepidoptera. ACS Synthetic Biology 2: 160-166. http://pubs.acs.org/doi/abs/10.1021/sb300123m

Jinek, M., K. Chylinski, I. Fonfara, M. Hauer, J. A. Doudna, and E. Charpentier. 2012. A programmable dual-RNA-guided DNA endonuclease in adaptive bacterial immunity. Science 337(6096): 816-821. https://www.ncbi.nlm.nih.gov/pubmed/22745249

Joga, M. R., M. J. Zotti, G. Smagghe, and O. Christiaens. 2016. RNAi efficiency, systemic properties, and novel delivery methods for pest insect control: what we know so far. Frontiers in Physiology 7: 11-13. https://doi.org/10.3389/fphys.2016.00553

Juan-Blasco, M., B. Sabater-Muñoz, R. Argilés, J. A. Jacas, P. Castañera, and A. Urbaneja. 2013. Molecular tools for sterile sperm detection to monitor Ceratitis capitata populations under SIT programmes. Pest Management Science 69: 857-864. https://doi.org/10.1002/ps.3448

Kaiser, P. E., J. A. Seawright, D. A. Dame, and D. J. Joslyn. 1978. Development of a genetic sexing system for Anopheles albimanus. J. Econ. Entomol. 71: 766-771. https://doi.org/10.1093/jee/71.5.766

Kalajdzic, P., and M. F. Schetelig. 2017. CRISPR/Cas-mediated gene editing using purified protein in Drosophila suzukii. Entomologia Experimentalis et Applicata 164: 350-362. http://onlinelibrary.wiley.com/doi/10.1111/eea.12599/abstract

KaramiNejadRanjbar, M., K. N. Eckermann, H. M. M. Ahmed, H. M. Sánchez C., S. Dippel, J. M. Marshall, and E. A. Wimmer. 2018. Consequences of resistance evolution in a Cas9-based sex conversion-suppression gene drive for insect pest management. Proceedings of the National Academy of Sciences of the USA 115(24): 6189-6194. https://doi.org/10.1073/pnas.1713825115

Kim, Y. G., J. Cha, and S. Chandrasegaran. 1996. Hybrid restriction enzymes: zinc finger fusions to Fok I cleavage domain. Proc. Nat. Acad. Sci. 93: 1156-1160. http://www.pnas.org/content/93/3/1156

Kistler, K. E., L. B. Vosshall, and B. J. Matthews. 2015. Genome engineering with CRISPR-Cas 9 in the mosquito Aedes aegypti. Cell Repts. 11: 51-60. https://www.ncbi.nlm.nih.gov/pubmed/25818303

Kiuchi, T., H. Koga, M. Kawamoto, K. Shoji, H. Sakai, Y. Arai, G. Ishihara, S. Kawaoka, S. Sugano, T. Shimada, Y. Suzuki, M. G. Suzuki, and S. Katsuma. 2014. A single female-specific piRNA is the primary determiner of sex in the silkworm. Nature 509(7502): 633-636. https://www.nature.com/articles/nature13315

Kleinstiver, B. P., V. Pattanayak, M. S. Prew, S. Q. Tsai, N. T. Nguyen, Z. Zheng, and J. K. Joung. 2016. High-fidelity CRISPR-Cas 9 nucleases with no detectable genome-wide off-target effects. Nature 529(7587):490-495. http://www.nature.com/nature/journal/v529/n7587/full/nature16526.html

Kokoza, V. A., and A. S. Raikhel. 2011. Targeted gene expression in the transgenic Aedes aegypti using the binary Gal4-UAS system. Insect Biochemistry and Molecular Biology 41: 637-644. https://www.ncbi.nlm.nih.gov/pubmed/21536128

Kolb, A. F. 2004. Genome engineering using site-specific recombinases. Cloning Stem Cells 4: 65-80. http://online.liebertpub.com/doi/abs/10.1089/153623002753632066

Krzywinska, E., and J. Krzywinski. 2018. Effects of stable ectopic expression of the primary sex determination gene Yob in the mosquito Anopheles gambiae. Parasites and Vectors 11(Suppl. 2): 648. https://doi.org/10.1186/s13071-018-3211-z 
Krzywinska, E., N. J. Dennison, G. J. Lycett, and J. Krzywinski. 2016. A maleness gene in the malaria mosquito Anopheles gambiae. Science 353(6294): 67-69. https://www.ncbi.nlm.nih.gov/pubmed/27365445

Kumar, P., S. S. Pandit, and I. T. Baldwin. 2012. Tobacco rattle virus vector: a rapid and transient means of silencing Manduca sexta genes by plant mediated RNA interference. PLOS ONE 7(2): e31347. http://journals.plos.org/plosone/article?id=10.1371/journal.pone.0031347

Kyritsis, G. A., A. A. Augustinos, I. Livadaras, C. Cáceres, K. Bourtzis, and N. T. Papadopoulos. 2019. Medfly-Wolbachia symbiosis: genotype $\mathrm{x}$ genotype interactions determine host's life history traits under mass rearing conditions. BMC Biotechnology 19(Suppl. 2): 96. https://doi.org/10.1186/s12896-019-0586-7

Labbé, G. M. C., D. D. Nimmo, and L. Alphey. 2010. piggybac- and PhiC31-mediated genetic transformation of the Asian tiger mosquito, Aedes albopictus (Skuse). PLOS Neglected Tropical Diseases 4(8):e788. http://journals.plos.org/plosntds/article?id=10.1371/journal.pntd.0000788

LaChance, L. E. 1967. The induction of dominant lethal mutations by ionizing radiation and chemicals as related to the sterile-male technique of insect control, pp. 617-650. In J. W. Wright and R. Pal (eds.), Genetics of insect vectors of disease. Elsevier. Amsterdam, The Netherlands.

Lagos, D., M. Koukidou, C. Savakis, and K. Komitopoulou. 2007. The transformer gene in Bactrocera oleae: the genetic switch that determines its sex fate. Insect Molecular Biology 16: 221230. http://onlinelibrary.wiley.com/doi/10.1111/j.1365-2583.2006.00717.x/abstract

Lebon, C., A. Benlali, C. Atyame, P. Mavingui, and P. Tortosa. 2018. Construction of a genetic sexing strain for Aedes albopictus: a promising tool for the development of sterilizing insect control strategies targeting the tiger mosquito. Parasites and Vectors 11(Suppl. 2): 658.

https://doi.org/10.1186/s13071-018-3212-y

Lee, G., and I. Saito. 1998. Role of nucleotide sequences of loxP spacer region in Cre-mediated recombination. Gene 216: 55-65. https://www.ncbi.nlm.nih.gov/pubmed/9714735

Lee, T., and L. Luo. 1999. Mosaic analysis with a repressible cell marker for studies of gene function in neuronal morphogenesis. Neuron 22: 451-461. https://www.ncbi.nlm.nih.gov/pubmed/10197526

Li, F., and M. J. Scott. 2016. CRISPR/Cas9-mediated mutagenesis of the white and Sex lethal loci in the invasive pest, Drosophila suzukii. Biochemical and Biophysical Research Communications 469: 911-916. https://www.ncbi.nlm.nih.gov/pubmed/26721433

Li, F., H. A. Wantuch, R. J. Linger, E. J. Belikoff, and M. J. Scott. 2014. Transgenic sexing system for genetic control of the Australian sheep blow fly Lucilia cuprina. Insect Biochemistry and Molecular Biology 51: 80-88. https://doi.org/10.1016/j.ibmb.2014.06.001

Li, J., and A. M. Handler. 2017. Temperature-dependent sex-reversal by a transformer-2 gene-edited mutation in the spotted wing drosophila, Drosophila suzukii. Scientific Reports 7(1): 12363. DOI: 10.1038/s41598-017-12405-4. https://www.nature.com/articles/s41598-017-12405-4

Li, T., S. Huang, W. Z. Jiang, D. Wright, M. H. Spalding, D. P. Weeks, and B. Yang. 2011. TAL nucleases (TALNs): hybrid proteins composed of TAL effectors and FokI DNA-cleavage domain. Nucleic Acids Research 39: 359-372. https://www.ncbi.nlm.nih.gov/pubmed/20699274

Lidholm, D. A., A. R. Lohe, and D. L. Hartl. 1993. The transposable element mariner mediates germline transformation in Drosophila melanogaster. Genetics 134: 859-868. http://www.genetics.org/content/134/3/859.long

Liu, J., C. Li, Z. Yu, P. Huang, H. Wu, C. Wei, N. Zhu, Y. Shen, Y. Chen, B. Zhang, W. M. Deng, and R. Jiao. 2012. Efficient and specific modifications of the Drosophila genome by means of an easy TALEN strategy. Journal of Genetics and Genomics 39: 209-215. https://www.ncbi.nlm.nih.gov/pubmed/22624882

Liu, P., B. Jin, X. Li, Y. Zhao, J. Gu, J. K. Biedler, Z. J. Tu, and X.-G. Chen. 2020. Nix is a maledetermining factor in the Asian tiger mosquito Aedes albopictus. Insect Biochemistry and Molecular Biology 118:103311. https://doi.org/10.1016/j.ibmb.2019.103311

Long, D-P., A-C. Zhao, X-J. Chen, Y. Zhang, W-J. Lu, Q. Guo, A. M. Handler, and Z-H. Xiang. 2012. FLP recombinase-mediated site-specific recombination in silkworm, Bombyx mori. PLOS ONE 7(6): e40150, 43: 997-1008. https://doi.org/10.1371/journal.pone.0040150

Long, D., A. Zhao, L. Xu, W. Lu, Q. Guo, Y. Zhang, and Z. Xiang. 2013. In vivo site-specific integration of transgene in silkworm via PhiC31 integrase-mediated cassette exchange. Insect Biochemistry \& Molecular Biology 43: 997-1008. https://www.ncbi.nlm.nih.gov/pubmed/23974010

Long, D., W. Lu, Y. Zhang, L. Bi, Z. Xiang, and A. Zhao. 2015. An efficient strategy for producing a stable, replaceable, highly efficient transgene expression system in silkworm, Bombyx mori. Scientific Reports 5: 8802. http://www.nature.com/articles/srep08802 
Loukeris, T. G., B. Arcà, I. Livadaras, G. Dialektaki, and C. Savakis. 1995. Introduction of the transposable element Minos into the germ line of Drosophila melanogaster. Proc. National Academy of Sciences of the USA 92: 9485-9489. https://www.ncbi.nlm.nih.gov/pubmed/7568159

Lynd, A., and G. J. Lycett. 2012. Development of the bi-partite Gal4-UAS system in the African malaria mosquito, Anopheles gambiae. PLOS ONE 7(2): e31552. https://doi.org/10.1371/journal.pone.0031552

Ma, S., J. Chang, X. Wang, Y. Liu, J. Zhang, W. Lu, J. Gao, R. Shi, P. Zhao, and Q. Xia. 2014. CRISPR/Cas9 mediated multiplex genome editing and heritable mutagenesis of BmKu70 in Bombyx mori. Scientific Reports 4: 4489. https://www.nature.com/articles/srep04489

Mao, Y. B., W. J. Cai, J. W. Wang, G. J. Hong, X. Y. Tao, L. J. Wang, Y. P. Huang, and X. Y. Chen. 2007. Silencing a cotton bollworm P450 monooxygenase gene by plant-mediated RNAi impairs larval tolerance of gossypol. Nature Biotechnology 25: 1307-1313. http://www.nature.com/nbt/journal/v25/n11/abs/nbt1352.html

Markert, M. J., Y. Zhang, M. S. Enuameh, S. M. Reppert, S. A. Wolfe, and C. Merlin. 2016. Genomic access to monarch migration using TALEN and CRISPR/Cas9-mediated targeted mutagenesis. G3 (Bethesda) 6(4): 905-915. https://www.ncbi.nlm.nih.gov/pubmed/26837953

Marois, E., C. Scali, J. Soichot, C. Kappler, E. A. Levashina, and F. Catteruccia. 2012. Highthroughput sorting of mosquito larvae for laboratory studies and for future vector control interventions. Malaria Journal 11: 302. https://doi.org/10.1186/1475-2875-11-302

Marraffini, L. A., and E. J. Sontheimer. 2010. CRISPR interference: RNA-directed adaptive immunity in bacteria and archaea. Nature Reviews Genetics 11: 181-190. http://www.nature.com/nrg/journal/v11/n3/full/nrg2749.html?foxtrotcallback=true

McArthur, C. C., J. M. Meredith, and P. Eggleston. 2014. Transgenic Anopheles gambiae expressing an antimalarial peptide suffer no significant fitness cost. PLOS ONE 9(2): e88625. https://doi.org/10.1371/journal.pone.0088625

McInnis, D. O., S. Tam, R. Lim, J. Komatsu, R. Kurashima, and C. Albrecht. 2004. Development of a pupal color-based genetic sexing strain of the melon fly, Bactrocera cucurbitae (Coquillett) (Diptera: Tephritidae). Annals of the Entomological Society of America 97: 1026-1033. https://doi.org/10.1603/0013-8746(2004)097[1026:DOAPCG]2.0.CO;2

Meccariello, A., S. M. Monti, A. Romanelli, R. Colonna, P. Primo, M. G. Inghilterra, G. Del Corsano, A. Ramaglia, G. Iazzetti, A. Chiarore, F. Patti, S. D. Heinze, M. Salvemini, H. Lindsay, E. Chiavacci, A. Burger, M. D. Robinson, C. Mosimann, D. Bopp, and G. Saccone. 2017. Highly efficient DNA-free gene disruption in the agricultural pest Ceratitis capitata by CRISPR-Cas9 ribonucleoprotein complexes. Scientific Reports 7: 10061. https://doi.org/10.1038/s41598-017-10347-5

Meccariello, A., M. Salvemini, P. Primo, B. Hall, P. Koskinioti, M. Dalíková, A. Gravina, M. A. Gucciardino, F. Forlenza, M.-E. Gregoriou, D. Ippolito, S. M. Monti, V. Petrella, M. M. Perrotta, S. Schmeing, A. Ruggiero, F. Scolari, E. Giordano, K. T. Tsoumani, F. Marec, N. Windbichler, K. P. Arunkumar, K. Bourtzis, K. D. Mathiopoulos, J. Ragoussis, L. Vitagliano, Z. Tu, P. A. Papathanos, M. D. Robinson, and G. Saccone. 2019. Maleness-on-the-Y (MoY) orchestrates male sex determination in major agricultural fruit fly pests. Science 365(6460): $1457-$ 1460. https://doi.org/10.1126/science.aax1318

Meredith, J. M., S. Basu, D. D. Nimmo, I. Larget-Thiery, E. L. Warr, A. Underhill, C. C. McArthur, V. Carter, H. Hurd, C. Bourgouin, and P. Eggleston. 2011. Site-specific integration and expression of an anti-malarial gene in transgenic Anopheles gambiae significantly reduces Plasmodium infections. PLOS ONE 6(1): e14587. https://doi.org/10.1371/journal.pone.0014587

Meredith, J. M., A. Underhill, C. C. McArthur, and P. Eggleston. 2013. Next-generation sitedirected transgenesis in the malaria vector mosquito Anopheles gambiae: self-docking strains expressing germline-specific phiC31 integrase. PLOS ONE 8(3): e59264. https://doi.org/10.1371/journal.pone.0059264

Meza, J. S., X. Nirmala, G. J. Zimowska, C. S. Zepeda-Cisneros, and A. M. Handler. 2011. Development of transgenic strains for the biological control of the Mexican fruit fly, Anastrepha ludens. Genetica 139: 53-62. https://www.ncbi.nlm.nih.gov/pubmed/20737195

Miles, A., N. J. Harding, G. Botta, C. Clarkson, T. Antao, K. Kozak, D. Schrider, A. Kern, S. Redmond, I. Sharakhov, R. Pearson, C. Bergey, M. Fontaine, A. Troco, A. Diabate, C. Costantini, K. Rohatgi, N. Elissa, B. Coulibaly, J. Dinis, J. Midega, C. Mbogo, H. Mawejje, J. Stalker, K. Rockett, E. Drury, D. Mead, A. Jeffreys, C. Hubbart, K. Rowlands, A. Isaacs, D. Jyothi, C. Malangone, P. Vauterin, B. Jeffrey, I. Wright, L. Hart, K. Kluczynski, V. Cornelius, 
B. MacInnis, C. Henrichs, R. Giacomantonio, D. Ayala, P. Bejon, N. Besansky, A. Burt, B. Caputo, A. della Torre, C. Godfray, M. Hahn, D. Neafsey, S. O'Loughlin, J. Pinto, M. Riehle, K. Vernick, D. Weetman, C. Wilding, B. White, M. Lawniczak, M. Donnelly, and D. Kwiatkowski. 2016. Natural diversity of the malaria vector Anopheles gambiae. https://doi.org/10.1101/096289 https://www.biorxiv.org/content/early/2016/12/22/096289

Missirlis, P. I., D. E. Smailus, and R. A. Holt. 2006. A high-throughput screen identifying sequence and promiscuity characteristics of the $\operatorname{lox} P$ spacer region in Cre-mediated recombination. BMC Genomics 7: 73. https://link.springer.com/article/10.1186/1471-2164-7-73

Mondal, K., A. G. Dastidar, G. Singh, S. Madhusudhanan, S. L. Gande, K. VijayRaghavan, and R. Varadarajan. 2007. Design and isolation of temperature-sensitive mutants of Gal4 in yeast and Drosophila. Journal of Molecular Biology 370: 939-950. https://doi.org/10.1016/j.jmb.2007.05.035

Moreira, L. A., J. Wang, F. H. Collins, and M. Jacobs-Lorena. 2004. Fitness of anopheline mosquitoes expressing transgenes that inhibit Plasmodium development. Genetics 166: 1337-1341. https://doi.org/10.1534/genetics.166.3.1337

Moreira, L. A., I. Iturbe-Ormaetxe, J. A. Jeffery, G. Lu, A. T. Pyke, L. M. Hedges, B. C. Rocha, S. Hall-Mendelin, A. Day, M. Riegler, L. E. Hugo, K. N. Johnson, B. H. Kay, E. A. McGraw, A. F. van den Hurk, P. A. Ryan, and S. L. O'Neill. 2009. A Wolbachia symbiont in Aedes aegypti limits infection with dengue, Chikungunya, and Plasmodium. Cell 139: 1268-1278. https://www.ncbi.nlm.nih.gov/pubmed/20064373

(NAPPO) North American Plant Protection Organization. 2007. Guidelines for importation and confined field release of transgenic arthropods in NAPPO member countries. NAPPO Regional Standards for Phytosanitary Measures (RSPM). RSPM No. 27. https:/www.nappo.org/files/1814/3753/9399/RSPM27-e.pdf

(NASEM) National Academies of Sciences, Engineering, and Medicine. 2016. Gene drives on the horizon: advancing science, navigating uncertainty, and aligning research with public values. https://www.nap.edu/catalog/23405/gene-drives-on-the-horizon-advancing-science-navigatinguncertainty-and

Nature. 2018. Foreign research funds, Australia's science minister and gene editing in Japan. The week in science: 24-30.08.18. Nature 560: 532-533. https://www.nature.com/articles/d41586-018-06041-9

Ndo, C., Y. Poumachu, D. Metitsi, H. P. Awono-Ambene, T. Tchuinkam, J. L. R. Gilles, and K. Bourtzis. 2018. Isolation and characterization of a temperature-sensitive lethal strain of Anopheles arabiensis for SIT-based application. Parasites and Vectors 11(Suppl. 2): 659. https://doi.org/10.1186/s13071-018-3216-7

Neel, J. V. 1983. Frequency of spontaneous and induced "point" mutations in higher eukaryotes. Journal of Heredity 74: 2-15. https://doi.org/10.1093/oxfordjournals.jhered.a109711

Nimmo, D. D., L. Alphey, J. M. Meredith, and P. Eggleston. 2006. High efficiency site-specific genetic engineering of the mosquito genome. Insect Molecular Biology 15: 129-136. http://onlinelibrary.wiley.com/doi/10.1111/j.1365-2583.2006.00615.x/full

Nirmala, X., S. R. Olson, T. C. Holler, K. H. Cho, and A. M. Handler. 2011. A DsRed fluorescent protein marker under polyubiquitin promoter regulation allows visual and amplified gene detection of transgenic Caribbean fruit flies in field traps. BioControl 56: 333-340. https://link.springer.com/article/10.1007/s10526-011-9347-9

Noble, C., J. Olejarz, K. M. Esvelt, G. M. Church, and M. A. Nowak. 2017. Evolutionary dynamics of CRISPR gene drives. Science Advances 3(4): e1601964. http://advances.sciencemag.org/content/3/4/e1601964

Nowak, C. M., S. Lawson, M. Zerez, and L. Bleris. 2016. Guide RNA engineering for versatile Cas9 functionality. Nucleic Acids Research 44: 9555-9564. https://doi.org/10.1093/nar/gkw908

Oberstein, A., A. Pare, L. Kaplan, and S. Small. 2005. Site-specific transgenesis by Cre-mediated recombination in Drosophila. Nature Methods 2: 583-585. https://www.ncbi.nlm.nih.gov/pubmed/16094382

O'Brochta, D. A., W. D. Warren, K. J. Saville, and P. W. Atkinson. 1996. Hermes, a functional nonDrosophilid insect gene vector from Musca domestica. Genetics 142: 907-914. https://www.ncbi.nlm.nih.gov/pubmed/8849896

Ogaugwu, C. E., M. F. Schetelig, and E. A. Wimmer. 2013. Transgenic sexing system for Ceratitis capitata (Diptera: Tephritidae) based on female-specific embryonic lethality. Insect Biochemistry and Molecular Biology 43: 1-8. https://doi.org/10.1016/j.ibmb.2012.10.010

Orban, P. C., D. Chui, and J. D. Marth. 1992. Tissue- and site-specific DNA recombination in transgenic mice. Proceedings of the National Academy of Sciences of the USA 89: 6861-6865. 
http://www.pnas.org/content/89/15/6861.abstract

Orozco, D., J. S. Meza, S. Zepeda, E. Solís, and J. L. Quintero-Fong. 2013. Tapachula-7, a new genetic sexing strain of the Mexican fruit fly (Diptera: Tephritidae): sexual compatibility and competitiveness. Journal of Economic Entomology 106: 735-741. https://doi.org/10.1603/EC12441

Pane, A., M. Salvemini, P. D. Bovi, C. Polito, and G. Saccone. 2002. The transformer gene in Ceratitis capitata provides a genetic basis for selecting and remembering the sexual fate. Development 129: 3715-3725. http://dev.biologists.org/content/129/15/3715.article-info

Pane, A., A. De Simone, G. Saccone, and C. Polito. 2005. Evolutionary conservation of Ceratitis capitata transformer gene function. Genetics 171: 615-624.

https:/www.ncbi.nlm.nih.gov/pmc/articles/PMC1456775/

Papanicolaou, A., M. F. Schetelig, P. Arensburger, P. W. Atkinson, J. B. Benoit, K. Bourtzis, P. Castañera, J. P. Cavanaugh, H. Chao, C. Childers, I. Curril, H. Dinh, H. V. Doddapaneni, A. Dolan, S. Dugan, M. Friedrich, G. Gasperi, S. Geib, G. Georgakilas, R. A. Gibbs, S. D. Giers, L. M. Gomulski, M. González-Guzmán, A. Guillem-Amat, Y. Han, A. G. Hatzigeorgiou, P. Hernández-Crespo, D. S. T. Hughes, J. W. Jones, D. Karagkouni, P. Koskinioti, S. L. Lee, A. R. Malacrida, M. Manni, K. Mathiopoulos, A. Meccariello, S. C. Murali, T. D. Murphy, D. M. Muzny, G. Oberhofer, F. Ortego, M. D. Paraskevopoulou, M. Poelchau, J. Qu, M. Reczko, H. M. Robertson, A. J. Rosendale, A. E. Rosselot, G. Saccone, M. Salvemini, G. Savini, P. Schreiner, F. Scolari, P. Siciliano, S. B. Sim, G. Tsiamis, E. Ureña, I. S. Vlachos, J. H. Werren, E. A. Wimmer, K. C. Worley, A. Zacharopoulou, S. Richards, and A. M. Handler. 2016. The whole genome sequence of the Mediterranean fruit fly, Ceratitis capitata (Wiedemann), reveals insights into the biology and adaptive evolution of a highly invasive pest species. Genome Biology 17: 192. https://doi.org/10.1186/s13059-016-1049-2

Parker, A., and K. Mehta. 2007. Sterile insect technique: a model for dose optimization for improved sterile insect quality. Florida Entomologist 90: 88-95. https://doi.org/10.1653/0015-4040(2007)90[88:SITAMF]2.0.CO;2

Patel, M. R., G. K. Miriyala, A. J. Littleton, H. Yang, K. Trinh, J. M. Young, S. R. Kennedy, Y. M. Yamashita, L. J. Pallanck, and H. S. Malik. 2016. A mitochondrial DNA hypomorph of cytochrome oxidase specifically impairs male fertility in Drosophila melanogaster. eLife 510.7554/eLife.16923 eLife 2016;5:e16923 https://doi.org/10.7554/eLife.16923.001

Pfeiffer, B. D., A. Jenett, A. S. Hammonds, T. T. B. Ngo, S. Misra, C. Murphy, A. Scully, J. W. Carlson, K. H. Wan, T. R. Laverty, C. Mungall, R. Svirskas, J. T. Kadonaga, C. Q. Doe, M. B. Eisen, S. E. Celniker, and G. M. Rubin. 2008. Tools for neuroanatomy and neurogenetics in Drosophila. Proc. Nat. Acad. Sci. USA 105: 9715-9720. http://www.pnas.org/content/105/28/9715

Phuc, H. K., M. H. Andreasen, R. S. Burton, C. Vass, M. J. Epton, G. Pape, G. Fu, K. C. Condon, S. Scaife, C. A. Donnelly, P. G. Coleman, H. White-Cooper, and L. Alphey. 2007. Late-acting dominant lethal genetic systems and mosquito control. BMC Biology 5:11. https://doi.org/10.1186/1741-7007-5-11

Pitino, M., A. D. Coleman, M. E. Maffei, C. J. Ridout, and S. A. Hogenhout. 2011. Silencing of aphid genes by dsRNA feeding from plants. PLOS ONE 6(10): e25709. https://doi.org/10.1371/journal.pone.0025709

Potter, C. J., B. Tasic, E. V. Russler, L. Liang, and L. Luo. 2010. The Q system: a repressible binary system for transgene expression, lineage tracing, and mosaic analysis. Cell 141: 536-548. https://www.ncbi.nlm.nih.gov/pubmed/20434990

Pridgeon, J. W., L. Zhao, J. J. Becnel, D. A. Strickman, G. G. Clark, and K. J. Linthicum. 2008. Topically applied AaeIAP ${ }_{1}$ double-stranded RNA kills female adults of Aedes aegypti. Journal of Medical Entomology 45: 414-420. https://doi.org/10.1093/jmedent/45.3.414

Pridgeon, J. W., L. Zhao, J. J. Becnel, D. A. Strickman, G. G. Clark, and K. J. Linthicum. 2016. Topically applied AaeIAP $P_{I}$ double-stranded RNA kills female adults of Aedes aegypti. Erratum. Journal of Medical Entomology 53(2): 484. https://doi.org/10.1093/jme/tjv192

Puglise, J. M., A. S. Estep, and J. J. Becnel. 2016. Expression profiles and RNAi silencing of inhibitor of apoptosis transcripts in Aedes, Anopheles, and Culex mosquitoes (Diptera: Culicidae). Journal of Medical Entomology 53: 304-314. https://doi.org/10.1093/jme/tjv191

Ramírez-Santos, E. M., P. Rendón, L. Ruiz-Montoya, J. Toledo, and P. Liedo. 2017a. Performance of a genetically modified strain of the Mediterranean fruit fly (Diptera: Tephritidae) for area-wide integrated pest management with the sterile insect technique. Journal of Economic Entomology 110: 24-34. https://doi.org/10.1093/jee/tow239 
Ramírez-Santos, E., P. Rendón, L. Ruiz-Montoya, J. Toledo, and P. Liedo. 2017b. Effect of irradiation doses on sterility and biological security in a genetically modified strain of the Mediterranean fruit fly (Diptera: Tephritidae). Journal of Economic Entomology 110: 1483-1494. https://doi.org/10.1093/jee/tox119

Rand, D. M., A. G. Clark, and L. M. Kann. 2001. Sexually antagonistic cytonuclear fitness interactions in Drosophila melanogaster. Genetics 159: 173-187. http://www.genetics.org/content/159/1/173

Ras, E., L. W. Beukeboom, C. Cáceres, and K. Bourtzis. 2017. Review of the role of gut microbiota in mass rearing of the olive fruit fly, Bactrocera oleae, and its parasitoids. Entomologia Experimentalis et Applicata 164: 237-256. http://onlinelibrary.wiley.com/doi/10.1111/eea.12609/full

Reeves, R. G., J. A. Denton, F. Santucci, J. Bryk, and F. A. Reed. 2012. Scientific standards and the regulation of genetically modified insects. PLOS Neglected Tropical Diseases 6(1): e1502. https://doi.org/10.1371/journal.pntd.0001502

Rempoulakis, P., G. Taret, I. U. Haq, V. Wornayporn, S. Ahmad, U. Sto Tomas, T. Dammalage, K. Gembinsky, G. Franz, C. Cáceres, and M. J. B. Vreysen. 2016. Evaluation of quality production parameters and mating behavior of novel genetic sexing strains of the Mediterranean fruit fly Ceratitis capitata (Wiedemann) (Diptera: Tephritidae). PLOS ONE 11(6): e0157679. https://doi.org/10.1371/journal.pone.0157679

Ren, X., J. Sun, B. E. Housden, Y. Hu, C. Roesel, S. Lin, L. P. Liu, Z. Yang, D. Mao, L. Sun, Q. Wu, J. Y. Ji, J. Xi, S. E. Mohr, J. Xu, N. Perrimon, and J. Q. Ni. 2013. Optimized gene editing technology for Drosophila melanogaster using germ line-specific Cas9. Proceedings of the National Academy of Sciences USA 110: 19012-19017. http://www.pnas.org/content/110/47/19012.short

Rendón, P., D. McInnis, D. Lance, and J. Stewart. 2004. Medfly (Diptera: Tephritidae) genetic sexing: large-scale field comparison of males-only and bisexual sterile fly releases in Guatemala. Journal of Economic Entomology 97: 1547-1553. https://doi.org/10.1603/0022-0493-97.5.1547

Riabinina, O., D. Task, E. Marr, C. C. Lin, R. Alford, D. A. O'Brochta, and C. J. Potter. 2016. Organization of olfactory centres in the malaria mosquito Anopheles gambiae. Nature Communications 7: 13010. https://www.nature.com/articles/ncomms13010

Robinson, G. E., K. J. Hackett, M. Purcell-Miramontes, S. J. Brown, J. D. Evans, M. R. Goldsmith, D. Lawson, J. Okamuro, H. M. Robertson, and D. J. Schneider. 2011. Creating a buzz about insect genomes. Science 331(6023): 1386. http://science.sciencemag.org/content/331/6023/1386

Rorth, P. 1998. Gal4 in the Drosophila female germline. Mechanisms of Development 78: 113-118. https://doi.org/10.1016/S0925-4773(98)00157-9

Rosen, L. E., H. A. Morrison, S. Masri, M. J. Brown, B. Springstubb, D. Sussman, B. L. Stoddard, and L. M. Seligman. 2006. Homing endonuclease I-CreI derivatives with novel DNA target specificities. Nucleic Acids Research 34(17): 4791-4800. https:/doi.org/10.1093/nar/gk1645

Saccone, G., M. Salvemini, and L. C. Polito. 2011. The transformer gene of Ceratitis capitata: a paradigm for a conserved epigenetic master regulator of sex determination in insects. Genetica 139: 99-111. https://link.springer.com/article/10.1007/s10709-010-9503-7

Sarkar, A., A. Atapattu, E. J. Belikoff, J. C. Heinrich, X. Li, C. Horn, E. A. Wimmer, and M. J. Scott. 2006. Insulated piggyBac vectors for insect transgenesis. BMC Biotechnology 6: 27. https://doi.org/10.1186/1472-6750-6-27

Sauer, B., and N. Henderson. 1988. Site-specific DNA recombination in mammalian cells by the Cre recombinase of bacteriophage P1. Proceedings of the National Academy of Sciences of the USA. 85: 5166-5170. https://www.ncbi.nlm.nih.gov/pmc/articles/PMC281709/

Schetelig, M. F., and A. M. Handler. 2012a. A transgenic embryonic sexing system for Anastrepha suspensa (Diptera: Tephritidae). Insect Biochemistry and Molecular Biology 42: 790-795. http://europepmc.org/abstract/med/22858603

Schetelig, M. F., and A. M. Handler. 2012b. Strategy for enhanced transgenic strain development for embryonic conditional lethality in Anastrepha suspensa. Proceedings of the National Academy of Sciences of the USA 109(24): 9348-9353. http://www.pnas.org/content/109/24/9348.abstract

Schetelig, M. F., and A. M. Handler. 2013a. A functional comparison of the $3 x P 3$ promoter by recombinase-mediated cassette exchange in Drosophila and a tephritid fly, Anastrepha suspensa. G3: Genes, Genomes, Genetics 3: 687-693. https://doi.org/10.1534/g3.112.005488

Schetelig, M. F., and A. M. Handler. 2013b. Y-linked markers for improved population control of the tephritid fruit fly pest, Anastrepha suspensa, pp. 123-133. In A. Vilcinskas (ed.), Yellow biotechnology II. Advances in biochemical engineering/biotechnology, vol. 136. Springer, Heidelberg, Germany. https://doi.org/10.1007/10_2013_209 
Schetelig, M. F., C. Cáceres, A. Zacharopoulou, G. Franz, and E. A. Wimmer. 2009a. Conditional embryonic lethality to improve the sterile insect technique in Ceratitis capitata (Diptera: Tephritidae). BMC Biology 7: 4. https://doi.org/10.1186/1741-7007-7-4

Schetelig, M. F., F. Scolari, A. M. Handler, S. Kittelmann, G. Gasperi, and E. A. Wimmer. 2009 b. Site-specific recombination for the modification of transgenic strains of the Mediterranean fruit fly Ceratitis capitata. Proceedings of the National Academy of Sciences of the USA 106: 18171-18176. http://www.pnas.org/content/106/43/18171.full

Schetelig, M. F., A. Milano, G. Saccone, and A. M. Handler. 2012. Male only progeny in Anastrepha suspensa by RNAi-induced sex reversion of chromosomal females. Insect Biochemistry and Molecular Biology 42: 51-57. https://doi.org/10.1016/j.ibmb.2011.10.007

Schetelig, M. F., A. Targovska, J. S. Meza, K. Bourtzis, and A. M. Handler. 2016. Tetracyclinesuppressible female lethality and sterility in the Mexican fruit fly, Anastrepha ludens. Insect Molecular Biology 25: 500-508. http://onlinelibrary.wiley.com/doi/10.1111/imb.12238/abstract

Schetelig, M. F., Y. Yan, Y. Zhao, and A. M. Handler. 2019. Genomic targeting by recombinasemediated cassette exchange in the spotted wing drosophila, Drosophila suzukii. Insect Molecular Biology 28(2): 187-195. https://doi.org/10.1111/imb.12537

Schinko, J. B., M. Weber, I. Viktorinova, A. Kiupakis, M. Averof, M. Klingler, E. A. Wimmer, and G. Bucher. 2010. Functionality of the GAL4/UAS system in Tribolium requires the use of endogenous core promoters. BMC Developmental Biology 10: 53. http://www.biomedcentral.com/1471-213X/10/53

Schlake, T., and J. Bode. 1994. Use of mutated FLP recognition target (FRT) sites for the exchange of expression cassettes at defined chromosomal loci. Biochemistry 33: 12746-12751. http://pubs.acs.org/doi/abs/10.1021/bi00209a003

Schliekelman, P., S. Ellner, and F. Gould. 2005. Pest control by genetic manipulation of sex ratio. Journal of Economic Entomology 98: 18-34. https://doi.org/10.1603/0022-0493-98.1.18

Schroeder, W. J., and W. C. Mitchell. 1981. Marking Tephritidae fruit fly adults in Hawaii for releaserecovery studies. Proc. Haw. Entomol. Soc. 23: 437-440. http://hdl.handle.net/10125/11129

Scolari, F., M. F. Schetelig, S. Bertin, A. R. Malacrida, G. Gasperi, and E. A. Wimmer. 2008. Fluorescent sperm marking to improve the fight against the pest insect Ceratitis capitata (Wiedemann; Diptera: Tephritidae). New Biotechnology 25: 76-84. https://www.ncbi.nlm.nih.gov/pubmed/18504022

Scott, M. J., C. Concha, J. B. Welch, P. L. Phillips, and S. R. Skoda. 2017. Review of research advances in the screwworm eradication program over the past 25 years. Entomologia Experimentalis et Applicata 164: 226-236. http://onlinelibrary.wiley.com/doi/10.1111/eea.12607/full

Sharma, A., S. D. Heinze, Y. Wu, T. Kohlbrenner, I. Morilla, C. Brunner, E. A. Wimmer, L. van de Zande, M. D. Robinson, L. W. Beukeboom, and D. Bopp. 2017. Male sex in houseflies is determined by $M d m d$, a paralog of the generic splice factor gene $C W C 22$. Science 356(6338): 642645. http://science.sciencemag.org/content/356/6338/642

Siegal, M. L., and D. L. Hartl. 1996. Transgene coplacement and high efficiency site-specific recombination with the Cre/loxP system in Drosophila. Genetics 144: 715-726. http://www.genetics.org/content/144/2/715

Siegal, M. L., and D. L. Hartl. 2000. Application of Cre/loxP in Drosophila. Site-specific recombination and transgene coplacement, pp. 487-495. In R. S. Tuan and C. W. Lo (eds.), Developmental biology protocols: Vol. II. Methods in molecular biology, vol. 136. Humana Press. https://doi.org/10.1385/1-59259-065-9:487

Simmons, G. S., A. R. McKemey, N. I. Morrison, S. O'Connell, B. E. Tabashnik, J. Claus, G. Fu, G. Tang, M. Sledge, A. S. Walker, C. E. Phillips, E. D. Miller, R. I. Rose, R. T. Staten, C. A. Donnelly, and L. Alphey. 2011. Field performance of a genetically engineered strain of pink bollworm. PLOS ONE 6(9): e24110. https://doi.org/10.1371/journal.pone.0024110

Smidler, A. L., O. Terenzi, J. Soichot, E. A. Levashina, and E. Marois. 2013. Targeted mutagenesis in the malaria mosquito using TALE nucleases. PLOS ONE 8(8): e74511. https://doi.org/10.1371/journal.pone.0074511

Smith, R. C., M. F. Walter, R. H. Hice, D. A. O'Brochta, and P. W. Atkinson. 2007. Testis-specific expression of the $\beta 2$ tubulin promoter of Aedes aegypti and its application as a genetic sex-separation marker. Insect Molecular Biology 16: 61-71. https://doi.org/10.1111/j.1365-2583.2006.0070

Smith, R. C., C. Kizito, J. L. Rasgon, and M. Jacobs-Lorena. 2013. Transgenic mosquitoes expressing a phospholipase $\mathrm{A}_{2}$ gene have a fitness advantage when fed Plasmodium falciparuminfected blood. PLOS ONE 8(10): e76097. https://doi.org/10.1371/journal.pone.0076097 
Sohal, D. S., M. Nghiem, M. A. Crackower, S. A. Witt, T. R. Kimball, K. M. Tymitz, J. M. Penninger, and J. D. Molkentin. 2001. Temporally regulated and tissue-specific gene manipulations in the adult and embryonic heart using a tamoxifen-inducible Cre protein. Circulation Research 89: 20-25. https://www.ncbi.nlm.nih.gov/pubmed/11440973

Spradling, A. C., D. M. Stern, I. Kiss, J. Roote, T. Laverty, and G. M. Rubin. 1995. Gene disruptions using P transposable elements: an integral component of the Drosophila genome project. Proc. Nat. Acad. Sci. USA 92: 10824-10830. http://www.pnas.org/content/92/24/10824

Sumitani, M., K. Kasashima, D. S. Yamamoto, K. Yagi, M. Yuda, H. Matsuoka, and S. Yoshida. 2013. Reduction of malaria transmission by transgenic mosquitoes expressing an antisporozoite antibody in their salivary glands. Insect Molecular Biology 22: 41-51. https://doi.org/10.1111/j.1365-2583.2012.01168.x

Sussman, D., M. Chadsey, S. Fauce, A. Engel, A. Bruett, R. Monnat, Jr., B. L. Stoddard, and L. M. Seligman. 2004. Isolation and characterization of new homing endonuclease specificities at individual target site positions. J. Mol. Biol. 342: 31-41. https://doi.org/10.1016/j.jmb.2004.07.031

Takasu, Y., I. Kobayashi, K. Beumer, K. Uchino, H. Sezutsu, S. Sajwan, D. Carroll, T. Tamura, and M. Zurovec. 2010. Targeted mutagenesis in the silkworm Bombyx mori using zinc finger nuclease mRNA injection. Insect Biochemistry and Molecular Biology 40: 759-765. https://www.ncbi.nlm.nih.gov/pubmed/20692340

Takasu, Y., S. Sajwan, T. Daimon, M. Osanai-Futahashi, K. Uchino, H. Sezutsu, T. Tamura, and M. Zurovec. 2013. Efficient TALEN construction for Bombyx mori gene targeting. PLOS ONE 8(9): e73458. https://doi.org/10.1371/journal.pone.0073458

Thomas, D. D., C. A. Donnelly, R. J. Wood, and L. S. Alphey. 2000. Insect population control using a dominant, repressible, lethal genetic system. Science 287(5462): 2474-2476. https://www.ncbi.nlm.nih.gov/pubmed/10741964

Thorpe, H. M., and M. C. M. Smith. 1998. In vitro site-specific integration of bacteriophage DNA catalyzed by a recombinase of the resolvase/invertase family. Proceedings of the National Academy of Sciences of the USA 95: 5505-5510. https://www.ncbi.nlm.nih.gov/pmc/articles/PMC20407/

Tian, H., H. Peng, Q. Yao, H. Chen, Q. Xie, B. Tang, and W. Zhang. 2009. Developmental control of a lepidopteran pest Spodoptera exigua by ingestion of bacteria expressing dsRNA of a non-midgut gene. PLOS ONE 4(7): e6225. https://doi.org/10.1371/journal.pone.0006225

Tobari, Y. N., and K. I. Kojima. 1972. A study of spontaneous mutation rates at ten loci detectable by starch gel electrophoresis in Drosophila melanogaster. Genetics 70: 397-403. http://www.genetics.org/content/70/3/397.short

Tsien, J. Z. 2016. Cre-lox neurogenetics: 20 years of versatile applications in brain research and counting. Frontiers in Genetics 7: 19. https://doi.org/10.3389/fgene.2016.00019

Turan, S., and J. Bode. 2011. Site-specific recombinases: from tag-and-target- to tag-and-exchangebased genomic modifications. FASEB Journal 25: 4088-4107. https://www.ncbi.nlm.nih.gov/pubmed/21891781

Turan, S., C. Zehe, J. Kuehle, J. Qiao, and J. Bode. 2013. Recombinase-mediated cassette exchange (RMCE) - a rapidly-expanding toolbox for targeted genomic modifications. Gene 515: 1-27. https://doi.org/10.1016/j.gene.2012.11.016

Turner, C. T., M. W. Davy, R. M. MacDiarmid, K. M. Plummer, N. P. Birch, and R. D. Newcomb. 2006. RNA interference in the light brown apple moth, Epiphyas postvittana (Walker) induced by double-stranded RNA feeding. Insect Molecular Biology 15: 383-391. https://doi.org/10.1111/j.1365-2583.2006.00656.x

Unckless, R. L., A. G. Clark, and P. W. Messer. 2017. Evolution of resistance against CRISPR/Cas9 gene drive. Genetics 207: 827-841. https://doi.org/10.1534/genetics.116.197285

Verhulst, E. C., L. van de Zande, and L. W. Beukeboom. 2010. Insect sex determination: it all evolves around transformer. Current Opinion in Genetics and Development 20: 376-383. https://doi.org/10.1016/j.gde.2010.05.001

Virginio, J. F., M. Gómez, A. M. Pinto, G. G. Aniely, B. J. Paranhos, C. A. T. Gava, C. Cáceres, and J. M. M. Walder. 2017. Male sexual competitiveness of two Ceratitis capitata strains, tsl Vienna 8 and OX3864A transgenics, in field cage conditions. Entomologia Experimentalis et Applicata 164: 318-326. http://onlinelibrary.wiley.com/doi/10.1111/eea.12615/full

Walker, T., P. H. Johnson, L. A. Moreira, I. Iturbe-Ormaetxe, F. D. Frentiu, C. J. McMeniman, Y. S. Leong, Y. Dong, J. Axford, P. Kriesner, A. L. Lloyd, S. A. Ritchie, S. L. O'Neill, and A. A. Hoffmann. 2011. The $w$ Mel Wolbachia strain blocks dengue and invades caged Aedes aegypti populations. Nature 476(7361): 450-453. 
http://www.nature.com/nature/journal/v476/n7361/full/nature10355.html?foxtrotcallback=true

Waltz, E. 2017. US government approves 'killer' mosquitoes to fight disease. Nature News 6 November. https://www.nature.com/news/us-government-approves-killer-mosquitoes-to-fight-disease-1.22959

Wang, Y., H. Zhang, H. Li, and X. Miao. 2011. Second-generation sequencing supply an effective way to screen RNAi targets in large scale for potential application in pest insect control. PLOS ONE 6(4): e18644. https://doi.org/10.1371/journal.pone.0018644

Wang, Y., Z. Li, J. Xu, B. Zeng, L. Ling, L. You, Y. Chen, Y. Huang, and A. Tan. 2013. The CRISPR/Cas system mediates efficient genome engineering in Bombyx mori. Cell Research 23: 1414-1416. http://www.nature.com/cr/journal/v23/n12/full/cr2013146a.html

Warren, W. D., P. W. Atkinson, and D. A. O'Brochta. 1994. The Hermes transposable element from the house fly, Musca domestica, is a short inverted repeat-type element of the hobo, Ac, and Tam3 (hAT) element family. Genetics Research 64: 87-97. https://doi.org/10.1017/S0016672300032699

White, D. J., J. N. Wolff, M. Pierson, and N. J. Gemmell. 2008. Revealing the hidden complexities of mtDNA inheritance. Molecular Ecology 17(23): 4925-4942. http://onlinelibrary.wiley.com/doi/10.1111/j.1365-294X.2008.03982.x/abstract

Whitten, M. M. A., P. D. Facey, R. Del Sol, L. T. Fernández-Martinez, M. C. Evans, J. J. Mitchell, O. G. Bodger, and P. J. Dyson. 2016. Symbiont-mediated RNA interference in insects. Proceedings of the Royal Society B Biological Sciences 283(1825): 20160042. http://rspb.royalsocietypublishing.org/content/283/1825/20160042

Whyard, S., A. D. Singh, and S. Wong. 2009. Ingested double-stranded RNAs can act as speciesspecific insecticides. Insect Biochemistry and Molecular Biology 39: 824-832. https://www.ncbi.nlm.nih.gov/pubmed/19815067

Wimmer, E. A. 2003. Innovations. Applications of insect transgenesis. Nature Reviews Genetics 4: 225-232. https://doi.org/10.1038/nrg1021 https://www.nature.com/articles/nrg1021

Wimmer, E. A. 2005. Insect transgenesis by site-specific recombination. Nature Methods 2: 580-582. https://doi.org/10.1038/nmeth0805-580

Windbichler, N., P. A. Papathanos, and A. Crisanti. 2008. Targeting the $X$ chromosome during spermatogenesis induces $\mathrm{Y}$ chromosome transmission ratio distortion and early dominant embryo lethality in Anopheles gambiae. PLOS Genetics 4(12): e1000291. https://doi.org/10.1371/journal.pgen.1000291

Windbichler, N., M. Menichelli, P. A. Papathanos, S. B. Thyme, H. Li, U. Y. Ulge, B. T. Hovde, D. Baker, R. J. Monnat, A. Burt, and A. Crisanti. 2011. A synthetic homing endonuclease-based gene drive system in the human malaria mosquito. Nature 473(7346): 212-215. http://www.nature.com/nature/journal/v473/n7346/full/nature09937.html

Wolff, J. N., D. M. Tompkins, N. J. Gemmell, and D. K. Dowling. 2016. Mitonuclear interactions, mtDNA-mediated thermal plasticity, and implications for the Trojan Female Technique for pest control. Scientific Reports 6: 30016. https://www.ncbi.nlm.nih.gov/pmc/articles/PMC4956753/

Wood, A. J., T. W. Lo, B. Zeitler, C. S. Pickle, E. J. Ralston, A. H. Lee, R. Amora, J. C. Miller, E. Leung, X. Meng, L. Zhang, E. J. Rebar, P. D. Gregory, F. D. Urnov, and B. J. Meyer. 2011. Targeted genome editing across species using ZFNs and TALENs. Science 333(6040): 307. https://www.ncbi.nlm.nih.gov/pubmed/21700836

Woodruff, R. C. 1992. Transposable DNA elements and life history traits. I. Transposition of P DNA elements in somatic cells reduces the lifespan of Drosophila melanogaster. Genetica 86: 143-154. https://www.ncbi.nlm.nih.gov/pubmed/1334906

Woodruff, R., B. Slatko, and J. Thompson. 1983. Factors affecting mutation rates in natural populations, pp. 37-124, 427-428. In M. Ashburner and H. L. Carson (eds.), The genetics and biology of Drosophila. Academic Press, London, UK.

Wynant, N., H. Verlinden, B. Breugelmans, G. Simonet, and J. Vanden Broeck. 2012. Tissuedependence and sensitivity of the systemic RNA interference response in the desert locust, Schistocerca gregaria. Insect Biochemistry and Molecular Biology 42: 911-917. http://dx.doi.org/10.1016/j.ibmb.2012.09.004

Wynant, N., D. Santos, R. Verdonck, J. Spit, P. Van Wielendaele, and J. Vanden Broeck. 2014. Identification, functional characterization and phylogenetic analysis of double stranded RNA degrading enzymes present in the gut of the desert locust, Schistocerca gregaria. Insect Biochemistry and Molecular Biology 46: 1-8. https:/www.ncbi.nlm.nih.gov/pubmed/24418314

Yamada, H., M. Q. Benedict, C. A. Malcolm, C. F. Oliva, S. M. Soliban, and J. R. L. Gilles. 2012. Genetic sex separation of the malaria vector, Anopheles arabiensis, by exposing eggs to dieldrin. Malaria Journal 11: 208. https://doi.org/10.1186/1475-2875-11-208 
Yan, Y., and M. J. Scott. 2015. A transgenic embryonic sexing system for the Australian sheep blow fly Lucilia cuprina. Scientific Reports 5: 16090. https://www.nature.com/articles/srep16090

Yee, W. K. W., K. L. Sutton, and D. K. Dowling. 2013. In vivo male fertility is affected by naturally occurring mitochondrial haplotypes. Current Biology 23: R55-R56. http://www.cell.com/current-biology/fulltext/S0960-9822(12)01442-X

Yu, Z., M. Ren, Z. Wang, B. Zhang, Y. S. Rong, R. Jiao, and G. Gao. 2013. Highly efficient genome modifications mediated by CRISPR/Cas9 in Drosophila. Genetics 195: 289-291. https://doi.org/10.1534/genetics.113.153825

Zacarés, M., G. Salvador-Herranz, D. Almenar, C. Tur, R. Argilés, K. Bourtzis, H. Bossin, and I. Pla. 2018. Exploring the potential of computer vision analysis of pupae size dimorphism for adaptive sex sorting systems of various vector mosquito species. Parasites and Vectors 11(Suppl. 2): 656. https://doi.org/10.1186/s13071-018-3221-x

Zha, W., X. Peng, R. Chen, B. Du, L. Zhu, and G. He. 2011. Knockdown of midgut genes by dsRNAtransgenic plant-mediated RNA interference in the hemipteran insect Nilaparvata lugens. PLOS ONE 6(5): e20504. https://doi.org/10.1371/journal.pone.0020504

Zhu, F., J. Xu, R. Palli, J. Ferguson, and S. R. Palli. 2011. Ingested RNA interference for managing the populations of the Colorado potato beetle, Leptinotarsa decemlineata. Pest Management Science 67: 175-182. http://onlinelibrary.wiley.com/doi/10.1002/ps.2048/abstract

Zimowska, G. J., X. Nirmala, and A. M. Handler. 2009. The beta2-tubulin gene from three tephritid fruit fly species and use of its promoter for sperm marking. Insect Biochemistry and Molecular Biology 39: 508-515. https://www.ncbi.nlm.nih.gov/pubmed/19520163 